Pacific

Journal of

Mathematics

VOGAN DIAGRAMS OF TWISTED AFFINE KAC-MOODY LIE ALGEBRAS

TANusRee PaL

Volume 239 No. 1

January 2009 


\title{
VOGAN DIAGRAMS OF TWISTED AFFINE KAC-MOODY LIE ALGEBRAS
}

\author{
TANUSREE PAL
}

\begin{abstract}
A Vogan diagram is a Dynkin diagram of a Kac-Moody Lie algebra of finite or affine type overlayed with additional structures. This paper develops the theory of Vogan diagrams for "almost compact" real forms of indecomposable twisted affine Kac-Moody Lie algebras and shows that equivalence classes of Vogan diagrams correspond to isomorphism classes of almost compact real forms of twisted affine Kac-Moody Lie algebras as given by H. Ben Messaoud and G. Rousseau.
\end{abstract}

\section{Introduction}

The classification of finite-dimensional real simple Lie algebras has been a classical problem. In 1914, Élie Cartan classified the simple Lie algebras over the reals for the first time. A number of subsequent simplifications of the proof followed until, in [1996b], A. W. Knapp derived a quick proof of Cartan's classification using the theory of Vogan diagrams.

The Kac-Moody Lie algebras are an infinite-dimensional generalization of the semisimple Lie algebras via the Cartan matrix and generators. The real forms of complex affine Lie algebras are of two kinds, "almost split" and "almost compact". Back-Valente, Bardy-Panse, Ben Messaoud and Rousseau [1995] classified the almost split real forms of the affine Kac-Moody Lie algebra, and Ben Messaoud and Rousseau [2003] gave a classification of the almost compact real forms. Work towards developing the theory of Vogan diagrams for the real forms of nontwisted affine Kac-Moody Lie algebras was done by P. Batra in [2000; 2002]. This paper develops the theory of Vogan diagrams for twisted affine Kac-Moody Lie algebras.

As introduced in [Knapp 1996a], a Vogan diagram is a Dynkin diagram of a Lie algebra with a diagram involution, such that the vertices fixed by the involution are either painted or unpainted depending on whether they are noncompact or compact. An important result in the theory of Vogan diagrams for real simple Lie algebras states first that any Vogan diagram can be transformed, by changing the ordering of its base, into a diagram that has at most one noncompact imaginary root and second

MSC2000: $17 \mathrm{~B} 67$.

Keywords: almost compact real forms, Vogan diagram, twisted affine Kac-Moody algebra. 
that that root occurs at most twice in the largest root of that simple Lie algebra. Since in the case of affine Kac-Moody algebras, changing the order does not give a Vogan diagram with at most one shaded root, Batra [2000] introduced a notion of equivalence of Vogan diagrams for nontwisted affine Kac-Moody Lie algebras. In this paper we modify the definition of the Vogan diagrams for the twisted affine Kac-Moody Lie algebras. In addition to the structural information already superimposed, a Vogan diagram now contains numerical labels on the vertices of the underlying Dynkin diagram; see Table 1. The classification of the almost compact real forms of affine Kac-Moody Lie algebras, as given in [Ben Messaoud and Rousseau 2003], prompts the definition of suitable equivalence relations among the Vogan diagrams for twisted affine Kac-Moody Lie algebras. With respect to this equivalence relation we prove the following result.

Theorem. Let $\mathfrak{g}$ be a twisted affine Kac-Moody Lie algebra. Then

- two almost compact real forms of $\mathfrak{g}$ having equivalent Vogan diagrams are isomorphic and

- every abstract Vogan diagram for $\mathfrak{g}$ represents an almost compact real form of $\mathfrak{g}$.

The analogues of these results for the nontwisted Kac-Moody Lie algebras were proved by Batra in [2000, Theorem 5.2] and [2002, Theorem 5.2], respectively. Owing to the difference in the structural realizations of the nontwisted and twisted affine Kac-Moody Lie algebras, the methods used in these two papers prove insufficient to yield the main theorems for the twisted affine Kac-Moody Lie algebras. This difficulty is resolved by using the notion of "adapted realization" of an affine Kac-Moody Lie algebra, as introduced in [Ben Messaoud and Rousseau 2003].

It is a fairly easy matter to work out representatives of the equivalence classes of Vogan diagrams for the twisted affine Kac-Moody Lie algebras. These have been listed in Tables 2 and 3. The equality of the number of nonequivalent Vogan diagrams and the number of nonisomorphic almost compact real forms as given in [Ben Messaoud and Rousseau 2003] seems to suggest the existence of a bijection between the equivalence classes of Vogan diagrams and the isomorphism classes of almost compact real forms of twisted affine Kac-Moody Lie algebras.

This paper is organized as follows. In Section 2, we review known facts about (complex) indecomposable twisted affine Kac-Moody Lie algebras $\mathfrak{g}$. In Section 3, we discuss the automorphisms and real forms of $\mathfrak{g}$, recall certain results from [Ben Messaoud and Rousseau 2003], which allow simple proofs of the main theorems, and study some properties of the Cartan subalgebras of the almost compact real forms of $\mathfrak{g}$. In Section 4, we introduce the Vogan diagrams, define their equivalence relations, and state and prove the main theorems. Tables 2 and 3 give the nonequivalent Vogan diagrams for the twisted affine Kac-Moody Lie algebras. 
Notation. The complexification $\mathfrak{m} \otimes \mathbb{C}$ of a real Lie algebra $\mathfrak{m}$ will be denoted by $\mathfrak{m}_{\mathfrak{C}}$. Given a finite order automorphism $\phi$ of a Lie algebra $\mathfrak{L}$, we shall denote by $\mathfrak{L}^{\phi}$ the fixed point subalgebra $\{x \in \mathfrak{L} \mid \phi(x)=x\}$ of $\mathfrak{L}$. By abuse of notation we shall denote $\mathbb{Z} \cap[m, n]$ by $[m, n]$ for all $m, n \in \mathbb{Z}$. For all integers $n, \varepsilon_{n}$ will denote the $n$-th root of unity.

\section{Kac-Moody Lie algebras}

2.1. Let $\mathfrak{g}$ be an affine Kac-Moody Lie algebra over the complex field $\mathbb{C}$. There exists a generalized Cartan matrix $A=\left(a_{i, j}\right)_{i, j \in[0, l]}$ such that $\mathfrak{g}=\mathfrak{g}(A)$ is generated by the Cartan subalgebra $\mathfrak{h}$ and the elements $e_{i}, f_{i}$ for $i \in[0, l]$; see [Kac 1990, Chapter 1]. We have a decomposition $\mathfrak{g}=\mathfrak{h} \oplus\left(\bigoplus_{\alpha \in \Delta} \mathfrak{g}_{\alpha}\right)$, where $\Delta \subset \mathfrak{h}^{*} \backslash\{0\}$ denotes the root system of $(\mathfrak{g}, \mathfrak{h})$. Let $\pi=\left\{\alpha_{i} \mid i \in[0, l]\right\}$ be the standard base of $\triangle$; let $\Delta_{+}=\triangle \cap\left(\bigoplus_{i \in[0, l]} \mathbb{N} \alpha_{i}\right)$ be the set of positive roots, and let $\Delta_{-}=-\Delta_{+}$be the set of negative roots of $\mathfrak{g}$. The coroots $\left(\alpha_{i}^{\vee}\right)_{i \in[0, l]} \subset \mathfrak{h}$ are such that $a_{i, j}=\alpha_{j}\left(\alpha_{i}^{\vee}\right)$ for $i, j \in[0, l]$. Let $W$ denote the Weyl group of $\mathfrak{g}$. We say $\alpha \in \Delta$ is a real root if $\alpha$ is $W$-conjugate to a root in $\pi$, and we denote the set of real roots by $\Delta^{\text {re }}$. The elements of $\Delta^{\mathrm{im}}=\Delta \backslash \Delta^{\mathrm{re}}$ are called the imaginary roots of $\mathfrak{g}$.

2.2. Realization of a Kac-Moody Lie algebra. Let $\dot{\mathfrak{g}}$ be a finite-dimensional simple Lie algebra over $\mathbb{C}$, let $\mu$ be a $k$-order automorphism of $\dot{\mathfrak{g}}$ for $k<\infty$, let $\varepsilon_{k}=e^{2 i \pi / k}$, a primitive $k$-th root of unity, and let $(\cdot, \cdot)$ be a nondegenerate, invariant, symmetric bilinear form on $\dot{\mathfrak{g}}$. For $j \in \mathbb{Z}_{k}$, let $\dot{\mathfrak{g}}_{j}=\left\{X \in \dot{\mathfrak{g}} \mid \mu(X)=\varepsilon_{k}^{j} X\right\}$; then $\dot{\mathfrak{g}}=\bigoplus_{j=0}^{k-1} \dot{\mathfrak{g}}_{j}$. By [Kac 1990], $(\dot{\mathfrak{g}})^{\mu}=\mathfrak{g}=\dot{\mathfrak{g}}_{0}$ is a simple finite-dimensional Lie algebra over $\mathbb{C}$. If $\dot{\mathfrak{h}}$ denotes the Cartan subalgebra of $\dot{\mathfrak{g}}$, then $\mathfrak{h}=\dot{\mathfrak{h}} \cap \dot{\mathfrak{g}}_{0}$ is the Cartan subalgebra of $\dot{\mathfrak{g}}_{0}$. We denote by $\mathfrak{g}$ the infinite-dimensional Lie algebra

$$
\mathfrak{l}\left(\dot{\mathfrak{g}}, \mu, \varepsilon_{k}\right)=\left(\bigoplus_{j \in \mathbb{Z}} \dot{\mathfrak{g}}_{(j \bmod k)} \otimes t^{j}\right) \oplus \mathbb{C} c \oplus \mathbb{C} d .
$$

The Lie algebra structure on $\mathfrak{g}$ is such that $c$ is the canonical central element and $\left[x \otimes t^{m}+\lambda d, y \otimes t^{n}+\lambda_{1} d\right]=\left([x, y] \otimes t^{m+n}+\lambda n y \otimes t^{n}-\lambda_{1} m x \otimes t^{m}\right)+m \delta_{m,-n}(x, y) c$, where $x, y \in \dot{\mathfrak{g}}$ and $\lambda, \lambda_{1} \in \mathbb{C}$. The element $d$ acts diagonally on $\mathfrak{g}$ with integer eigenvalues and induces $\mathbb{Z}$-gradation on $\mathfrak{l}\left(\dot{\mathfrak{g}}, \mu, \varepsilon_{k}\right)$. The Lie algebra $\mathfrak{l}(\dot{\mathfrak{g}}, \mathrm{Id}, 1)$ with $\mu=\mathrm{Id}$ denotes a nontwisted affine Kac-Moody Lie algebra, and $\mathfrak{l}\left(\dot{\mathfrak{g}}, \mu, \varepsilon_{k}\right)$ for $\mu \neq \mathrm{Id}$ and $k=2$ or 3 denotes a twisted affine Kac-Moody Lie algebra. Clearly $\mathfrak{g}^{\prime \prime}=\mathfrak{l}\left(\dot{\mathfrak{g}}, \mu, \varepsilon_{k}\right)^{\prime \prime}=\bigoplus_{j \in \mathbb{Z}} \dot{\mathfrak{g}}_{(j \bmod k)} \otimes t^{j}$ is the fixed point set of the automorphism $\tilde{\mu}$ of $\mathfrak{l}(\dot{\mathfrak{g}}, \mathrm{Id}, 1)^{\prime \prime}=\dot{\mathfrak{g}} \otimes \mathbb{C}\left[t, t^{-1}\right]$ defined by

$$
\tilde{\mu}\left(x \otimes t^{j}\right)=\left(\epsilon_{k}\right)^{j} \mu(x) \otimes t^{j} \quad \text { for } j \in \mathbb{Z} \text { and } x \in \dot{\mathfrak{g}} .
$$

Also $\mathfrak{h}=\mathfrak{h} \otimes 1 \oplus \mathbb{C} c \oplus \mathbb{C} d$ is the standard Cartan subalgebra of $\mathfrak{g}$. 


\begin{tabular}{|c|c|c|c|}
\hline Type Aff $k$ & $\mathfrak{g}$ & Dynkin diagram & $\stackrel{\circ}{\mathfrak{g}}$ \\
\hline 2 & $A_{2}^{(2)}$ & 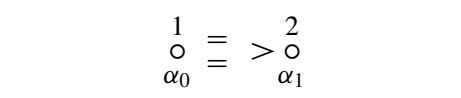 & $A_{1}$ \\
\hline 2 & $A_{2 l}^{(2)}(l \geq 2)$ & $\underset{\alpha_{0}}{\stackrel{1}{\circ}} \underset{\alpha_{1}}{\stackrel{\circ}{\circ}}-\cdots-\underset{\alpha_{l-1}}{\stackrel{\circ}{\circ}} \Rightarrow \underset{\alpha_{l}}{\stackrel{\circ}{\circ}}$ & $B_{l}$ \\
\hline 2 & $A_{2 l-1}^{(2)}(l \geq 3)$ & 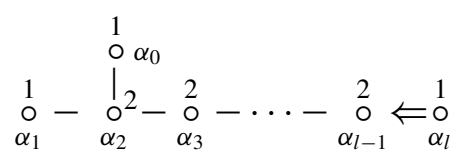 & $C_{l}$ \\
\hline 2 & $D_{l+1}^{(2)}(l \geq 2)$ & 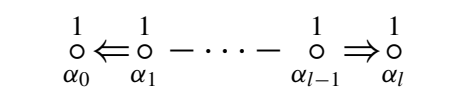 & $B_{l}$ \\
\hline 2 & $E_{6}^{(2)}$ & 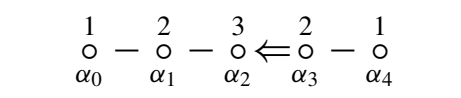 & $F_{4}$ \\
\hline 3 & $D_{4}^{3}$ & $\underset{\alpha_{2}}{1}-\underset{\alpha_{1}}{\stackrel{2}{\circ}} \Leftarrow \underset{\alpha_{2}}{\stackrel{1}{\circ}}$ & $G_{2}$ \\
\hline
\end{tabular}

Table 1. Dynkin diagrams of the twisted affine Kac-Moody Lie algebras.

2.3. Let $\left(\dot{e}_{i}, \dot{f}_{i}\right)_{i=1, \ldots, n}$ be a system of Chevalley generators of $\dot{\mathfrak{g}}$. The simple coroots, $\dot{\alpha}_{i}^{\vee}=\left[\dot{e}_{i}, \dot{f}_{i}\right]$ for $i=1, \ldots, n$, form a base of $\dot{\mathfrak{h}}$. Let $\dot{\omega}$ be the Cartan involution of $\dot{\mathfrak{g}}$ given by $\dot{\omega}\left(\dot{e}_{i}\right)=-\dot{f}_{i},\left.\dot{\omega}\right|_{\mathfrak{h}}=-$ Id and $\dot{\omega}^{2}=$ Id. The simple roots in the base $\dot{\pi}$ of $\dot{\mathfrak{g}}$ are enumerated in such a way that a system of representatives of the $\mu$-orbits of $\{1, \ldots, n\}$ is $\{1, \ldots, l\}$. Since the order of $\mu(\neq \mathrm{Id})$ is $k(=2$ or 3$)$, the cardinality $n_{i}$ of the $\mu$-orbit of $\dot{\alpha}_{i}^{\vee}$ is either 1 or $k$ for any $i \in\{1, \ldots, n\}$. Correspondingly, $e_{i}=\dot{e}_{i}+\cdots+\mu^{n_{i}-1}\left(\dot{e}_{i}\right)$ except in the case of $A_{2 l}^{(2)}$, where $e_{l}=\sqrt{2}\left(\dot{e}_{l}+e_{l+1}\right)$, $f_{i}=\stackrel{\circ}{\omega}\left(\dot{e}_{i}\right)$ and $\alpha_{i}^{\vee}=\left[e_{i}, f_{i}\right]$. Hence, $\left(e_{i}, f_{i}\right)_{i=1, \ldots, l}$ is a system of Chevalley generators of the simple Lie algebra $\mathfrak{\mathfrak { g }}$. Let $\theta_{0} \in(\mathfrak{h})^{*}$ be the highest weight of the irreducible $\mathfrak{\mathfrak { g }}$-module $\dot{\mathfrak{g}}_{1}$. Choose $E_{0} \in\left(\dot{\mathfrak{g}}_{1}\right)_{-\theta_{0}}$ and put $F_{0}=-\dot{\omega}\left(E_{0}\right), e_{0}=E_{0} \otimes t$, $f_{0}=F_{0} \otimes t^{-1}$ and $\alpha_{0}^{\vee}=\left[e_{0}, f_{0}\right]$. Then $\left\{\mathfrak{h}, e_{i}, f_{i}, i \in[0, l]\right\}$ is a system of generators of $\mathfrak{g}(A)$, the Lie algebra associated to a generalized Cartan matrix $A$; see [Kac 1990, Theorem 8.3].

A compact form $\mathfrak{u}(A)$ of $\mathfrak{g}(A)$ is defined as the fixed point set of $\mathfrak{g}(A)$ under the compact involution $\omega$ defined on $\dot{\mathfrak{g}} \otimes \mathbb{C}\left[t, t^{-1}\right]$ as follows: $\omega\left(x \otimes t^{j}\right)=\dot{\omega}(x) \otimes t^{-j}$.

2.4. A graph $S(A)$ called the Dynkin diagram of $A$ can be associated to a generalized Cartan matrix (GCM) $A$ as explained in [Kac 1990]. A Dynkin diagram $S(\AA)$ is obtained from $S(A)$ by removing the 0 -th vertex. The corresponding GCM $\AA$ is of finite type, and $\mathfrak{g}(\AA)=\mathfrak{g}$ is a simple finite-dimensional Lie algebra. It is clear that $A$ is indecomposable if and only if $S(A)$ is a connected graph. The matrix $A$ is determined by the Dynkin diagram and the enumeration of its vertices. Table 1 
gives the Dynkin diagrams of the twisted affine Kac-Moody Lie algebras. The enumeration of the generators is the same as in [Kac 1990] except in the case $A_{2 l}^{(2)}$, where the enumeration is reversed and the case $A_{2}^{(2)}$, where the Dynkin diagram and enumeration has been taken from [Moody and Pianzola 1995, Section 3.5].

2.5. Let $\triangle$ be the root system of $\stackrel{\mathfrak{g}}{\text {. Let }} \stackrel{\circ}{\pi}=\left(\alpha_{i}\right)_{i \in[1, l]}$ be a base of $(\stackrel{\mathfrak{g}}{\mathfrak{h}} \mathfrak{h})$, and let $\left(\stackrel{\circ}{p}_{i}\right)_{i \in[1, l]}$ be the corresponding dual basis in $\mathfrak{h}$. Denote by $\AA_{s}, \AA_{l}$ and $\AA_{+}$the short, long and positive roots of $\mathfrak{g}$. Define an element $\delta \in \mathfrak{h}^{*}$ by putting $\delta(d)=1$ and $\delta(\mathfrak{h}+\mathbb{C} c)=0$. Recall from [Kac 1990, Proposition 6.3] the following description of $\triangle^{\text {re }}$ for $\mathfrak{g}$ not of type $A_{2 l}^{(2)}$ :

$$
\triangle^{\text {re }}=\left\{\alpha+n \delta \mid \alpha \in \AA_{s}, n \in \mathbb{Z}\right\} \cup\left\{\alpha+n k \delta \mid \alpha \in \AA_{l}, n \in \mathbb{Z}\right\} \quad \text { if } k=2 \text { or } 3 .
$$

With respect to the enumeration of the simple roots of $A_{2 l}^{(2)}$ as given in Table 1, calculations similar to those in [Kac 1990, Proposition 6.3] show that for $\mathfrak{g}$ of type $A_{2 l}^{(2)}$, we have $\triangle^{\mathrm{re}}=\triangle_{s}^{\mathrm{re}} \cup \triangle_{m}^{\mathrm{re}} \cup \triangle_{l}^{\mathrm{re}}$, where

$$
\begin{aligned}
& \triangle_{s}^{\mathrm{re}}=\left\{\frac{1}{4}(2 \alpha+(4 n-k) \delta) \mid \alpha \in \AA_{l}, n, k \in \mathbb{Z}, 1 \leq k \leq 3\right\}, \\
& \triangle_{m}^{\mathrm{re}}=\left\{\frac{1}{2}(2 \alpha+(2 n-1) \delta) \mid \alpha \in \AA_{l}, n \in \mathbb{Z}\right\}, \\
& \triangle_{l}^{\mathrm{re}}=\left\{2 \alpha+n \delta \mid \alpha \in \triangle_{l}, n \in \mathbb{Z}\right\} .
\end{aligned}
$$

Let $\triangle_{+}^{\mathrm{re}}=\left\{\alpha \in \triangle^{\mathrm{re}}\right.$ with $\left.n>0\right\} \cup \triangle_{+}$. By [Kac 1990, Section 6.4, Proposition 6.4], $a_{0} \alpha_{0}=\delta-\theta$ with $\theta \in\left(\triangle_{+}\right)_{s}$ for $\mathfrak{g}$ of type Aff 2 or 3 and not of type $A_{2 l}^{(2)}$, and $\alpha_{0}=\delta-2 \theta$ with $\theta \in\left(\AA_{+}\right)_{l}$ for $\mathfrak{g}$ of type $A_{2 l}^{(2)}$. If $a_{0}, a_{1}, \ldots, a_{l}$ are the numerical labels of $S(A)$ as in Table 1 , then the element $\delta \in \mathfrak{h}^{*}$ is defined as $\delta=\sum_{i=0}^{l} a_{i} \alpha_{i}$, and we have $\triangle^{\mathrm{im}}=\{ \pm \delta, \pm 2 \delta, \ldots\}$ and $\triangle_{+}^{\mathrm{im}}=\{\delta, 2 \delta, \ldots\}$. The set $\Pi=\left(\alpha_{i}\right)_{i=0,1, \ldots, l}$ is a base of $\triangle$. Setting $p_{0}=d$ and $p_{i}=\stackrel{\circ}{p}_{i}+a_{i} d$ for $i=1, \ldots, l$, we obtain a family $\left(p_{i}\right)_{i \in[0, l]} \subset \mathfrak{h}$ satisfying $\alpha_{j}\left(p_{i}\right)=\delta_{i, j}$ for $i, j \in[0, l]$.

Let $\mathfrak{g}_{\gamma}$ be the root space of $\gamma \in \triangle$. For $\gamma \in \triangle^{\mathrm{re}}, \operatorname{dim} \mathfrak{g}_{\gamma}=1$.

For a root $\dot{\alpha}$ of $\dot{\mathfrak{g}}$, if $\dot{e}_{ \pm \dot{\alpha}} \in \dot{\mathfrak{g}}_{\dot{\alpha}}$ is such that the $\mathbb{C}$-span of $\left\{\dot{e}_{\dot{\alpha}}, \dot{e}_{-\dot{\alpha}}, H_{\dot{\alpha}}=\left[\dot{e}_{\dot{\alpha}}, \dot{e}_{-\dot{\alpha}}\right]\right\}$ is isomorphic to $\mathfrak{s l}_{2}$, then, given $\alpha+n k \delta \in \Delta^{\text {re }}$ with $\alpha \in \check{\triangle}_{l}$,

$$
E_{ \pm \alpha, \pm k s}=e_{ \pm \alpha} \otimes t^{ \pm k s} \in \mathfrak{g}_{ \pm(\alpha+k s)}
$$

and $H_{\alpha} \in \mathfrak{h}$ can be chosen so that the $\mathbb{C}$-span of $\left\{E_{\alpha, k s}, H_{\alpha}, E_{-\alpha,-k s}\right\}$ is isomorphic to $\mathfrak{s l}_{2}$. If $\alpha \in \AA_{s}$, then there exists $\dot{\alpha} \in \dot{\mathfrak{g}}$ such that for $j \in \mathbb{Z}_{k}$ one can choose

$$
\begin{aligned}
E_{ \pm \alpha, \pm k s+j} & =\left(\varepsilon_{k}^{j} e_{ \pm \mu(\dot{\alpha})}+\cdots+\varepsilon_{k}^{j k} e_{ \pm \mu^{k}(\dot{\alpha})}\right) \otimes t^{ \pm k s+j} \in \mathfrak{g}_{ \pm(\alpha+k s+j)}, \\
h_{\alpha}^{j} & =\varepsilon_{k}^{j} H_{\mu(\dot{\alpha})}+\cdots+\varepsilon_{k}^{j k} H_{\mu^{k}(\dot{\alpha})} \in \dot{\mathfrak{h}}_{j}
\end{aligned}
$$

such that the $\mathbb{C}$-span of $\left\{E_{\alpha, k s+j}, h_{\alpha}^{j}, E_{-\alpha,-k s-j}\right\}$ is isomorphic to $\mathfrak{s h}_{2}$. 
2.6. Let $\stackrel{\circ}{W}$ be the Weyl group of $\mathfrak{g}$ generated by the reflections $\left(r_{i}\right)_{i \in[1, l]}$. Let $T$ be the group of translations. By [Kac 1990, Proposition 6.5], $W=\stackrel{\circ}{W} \ltimes T$ is the Weyl group of $\mathfrak{g}$. Since the Weyl chambers are in one to one correspondence with bases of a finite root system and $w(\delta)=\delta$ for all $w \in W$, it follows from [Kac 1990, Section 2.7 and Proposition 3.12(b)]Kac that given two positive root systems $\triangle_{+}$and $\triangle_{+}^{\prime}$ of a compact form $\mathfrak{u}(A)$ of $\mathfrak{g}(A)$, there exists an $s \in W$ such that $s . \triangle_{+}^{\prime}=\triangle_{+}$. But for $\alpha \in \triangle$, the generators $r_{\alpha}$ of $\stackrel{\circ}{W}$ are interior automorphisms; see [Ben Messaoud and Rousseau 2003, Lemma 5.3]. Hence given two positive root systems $\triangle_{+}$and $\triangle_{+}^{\prime}$ of $\mathfrak{u}(A)$, there exists an $s \in \operatorname{Int}(\mathfrak{u}(A))$ such that $s . \triangle_{+}^{\prime}=\triangle_{+}$.

\section{Automorphisms and real forms of $\mathfrak{g}$}

3.1. Define a group $G$ acting on $\mathfrak{g}$ by the adjoint representation $\operatorname{Ad}: G \rightarrow \operatorname{Aut}(\mathfrak{g})$. It is generated by the subgroups $U_{\alpha}$, for $\alpha \in \triangle^{\text {re }}$, that are isomorphic to the additive groups $\mathfrak{g}_{\alpha}$ by an isomorphism exp such that $\operatorname{Ad} \circ \exp =\exp \circ \mathrm{ad}$.

A Cartan subalgebra (CSA) of $\mathfrak{g}$ is a maximal $a_{\mathfrak{g}}$-diagonalizable Lie subalgebra. The CSAs are all conjugate by G. A Borel subalgebra (BSA) is a completely solvable maximal subalgebra of $\mathfrak{g}$. The subalgebras $\mathfrak{b}^{+}=\mathfrak{h} \oplus\left(\bigoplus_{\alpha \in \Delta^{+}} \mathfrak{g}_{\alpha}\right)$ and $\mathfrak{b}^{-}=\mathfrak{h} \oplus\left(\bigoplus_{\alpha \in \Delta^{-}} \mathfrak{g}_{\alpha}\right)$ are respectively called the positive and negative standard BSAs. The subalgebras $\mathfrak{b}^{+}$and $\mathfrak{b}^{-}$are not conjugate by G. All the BSAs conjugate to $\mathfrak{b}^{+}$(respectively $\mathfrak{b}^{-}$) are said to be positive (respectively negative). If $\mathfrak{g}$ is indecomposable, all BSAs are either positive or negative.

An automorphism (linear or semilinear) of $\mathfrak{g}$ acts in a compatible manner to Ad on $G$ and hence transforms two conjugate BSAs to two conjugate BSAs; it is said to be of first type (respectively second type) if it transforms a positive BSA to a positive (respectively negative) BSA. If $\mathfrak{g}$ is indecomposable, all automorphisms are either of first or second type.

3.2. Automorphisms of $\mathfrak{g}$. By [Rousseau 1989], the group of automorphisms of $\mathfrak{g}$ is given by $\operatorname{Aut}(\mathfrak{g})=[(\{1, \omega\} \times \operatorname{Aut}(A)) \ltimes \operatorname{Int}(\mathfrak{g})] \ltimes \operatorname{Tr}$, where $\omega$ is the Cartan involution of $\mathfrak{g}, \operatorname{Aut}(A)$ is the group of permutations of $[0, l]$ such that $a_{\rho i, \rho j}=a_{i j}$ for $1 \leq i, j \in l, \operatorname{Int}(\mathfrak{g})$ is the set of interior automorphisms of $\mathfrak{g}$, and $\operatorname{Tr}=\operatorname{Tr}\left(\mathfrak{g}, \mathfrak{g}^{\prime}, \mathfrak{c}\right)$ is the group of transvections of $\mathfrak{g}$ as defined in [Rousseau 1989, 2.4].

Let $\mathfrak{h}$ be a standard CSA of $\mathfrak{g}$. A group $\widetilde{H}$ is defined such that, in the complex case, $\operatorname{Ad}(\widetilde{H})=\exp \operatorname{ad}(\mathfrak{h})$; see [Peterson and $\operatorname{Kac} 1983]$. The $\operatorname{group} \operatorname{Int}(\mathfrak{g})=$ $\operatorname{Ad}(\widetilde{H} \ltimes G)$ of interior automorphisms of $\mathfrak{g}$ is the image of the semidirect product of $\widetilde{H}$ and $G$. Its derived group is the adjoint $\operatorname{group} \operatorname{Ad}(G)$ and is denoted by $\operatorname{Int}\left(\mathfrak{g}^{\prime}\right)$. Since $G$ acts transitively on the Cartan subalgebra, the group $\operatorname{Int}\left(\mathfrak{g}^{\prime}\right)$ does not depend on the choice of $\mathfrak{h}$. 
Definition. Let $\operatorname{Aut}_{\mathbb{R}}(\mathfrak{g})$ denote the group of automorphisms of $\mathfrak{g}$ that are either $\mathbb{C}$-linear or semilinear, that is, $\phi(\lambda x)=\bar{\lambda} \phi(x)$ for all $\lambda \in \mathbb{C}$ and $x \in \mathfrak{g}$. Aut $(\mathfrak{g})$ is an index 2 normal subgroup of $\operatorname{Aut}_{\mathbb{R}}(\mathfrak{g})$.

A semiinvolution of $\mathfrak{g}$ is a semilinear automorphism of order 2. For all semiinvolutions $\sigma^{\prime}$ we have a decomposition $\operatorname{Aut}_{\mathbb{R}}(\mathfrak{g})=\left\{1, \sigma^{\prime}\right\} \ltimes \operatorname{Aut}(\mathfrak{g})$. If $\sigma^{\prime}$ is a semiinvolution of $\mathfrak{g}$, the real Lie algebra $\mathfrak{g}_{\mathbb{R}}=\mathfrak{g}^{\sigma^{\prime}}$ is a real form of $\mathfrak{g}$ in the sense that there exists an isomorphism of the complex Lie algebras $\mathfrak{g}_{\mathbb{R}} \otimes_{\mathbb{C}} \mathbb{C}$ and $\mathfrak{g}$. Further, $\sigma^{\prime}$ is the conjugation of $\mathfrak{g}$ with respect to $\mathfrak{g}_{\mathbb{R}}$. Thus there exists a bijection between the semiinvolutions and real forms. The standard normal (or split) real form of $\mathfrak{g}$ is the real Lie algebra generated by $e_{i}, f_{i}, \alpha_{i}^{\vee}$ and $d$. The corresponding semiinvolution $\sigma_{n}^{\prime}$ is called the normal semiinvolution. Note that $\sigma_{n}^{\prime}$ is the restriction of $\dot{\sigma}_{n}^{\prime} \otimes$ conj on $\mathfrak{g}^{\prime \prime}=\left(\dot{\mathfrak{g}} \otimes \mathbb{C}\left[t, t^{-1}\right]\right)^{\tilde{\mu}}$, where $\dot{\sigma}_{n}^{\prime}$ is the normal semiinvolution of $\dot{\mathfrak{g}}$ and $\operatorname{conj}(P(t))=\bar{P}(t)$, and $\sigma_{n}^{\prime}$ commutes with the standard Cartan involution $\omega$.

The standard Cartan semiinvolution $\omega^{\prime}$ of $\mathfrak{g}$ is the unique semiinvolution of $\mathfrak{g}$ such that $\omega^{\prime}\left(e_{i}\right)=-f_{i}$ and $\omega^{\prime}(d)=-d$. Hence $\omega^{\prime}=\sigma_{n}^{\prime} \omega=\omega \sigma_{n}^{\prime}$. In the standard realization of $\mathfrak{g}, \omega^{\prime}$ induces on $\mathfrak{g}^{\prime \prime}$ the restriction of $\dot{\omega} \otimes \iota^{\prime}$, where $\dot{\omega}$ is the Cartan semiinvolution of $\dot{\mathfrak{g}}$ and $\iota^{\prime}(P(t))=\bar{P}\left(t^{-1}\right)$. All conjugates of $\omega^{\prime}$ are called Cartan semiinvolutions (CSI) or compact semiinvolutions; these are semiinvolutions of the second type. The corresponding real forms are called the compact real forms. It is clear that, for all affine Kac-Moody Lie algebras, there exists, up to a conjugation, a unique compact real form.

3.3. Real forms of $\mathfrak{g}$. The real form corresponding to a semiinvolution of first type (SI1) (respectively of second type (SI2)) is said to be almost split (respectively almost compact) real form. Up to a conjugation, a classification of the almost split real forms was given in [Back-Valente et al. 1995] and a classification of the almost compact real forms was given in [Ben Messaoud and Rousseau 2003].

3.4. Cartan subalgebra of a real form of $\mathfrak{g}$. Let $\mathfrak{g}_{\mathbb{R}}$ be a real form of the complex Lie algebra $\mathfrak{g}$. A Lie subalgebra $\mathfrak{h}_{0}$ of $\mathfrak{g}_{\mathbb{R}}$ is called the Cartan subalgebra of $\mathfrak{g}_{\mathbb{R}}$ if the complexification, $\mathfrak{h}_{0} \otimes \mathbb{C}$, is a Cartan subalgebra of $\mathfrak{g}$.

3.5. Cartan involutions. Let $\sigma^{\prime}$ be an SI 2 of $\mathfrak{g}$, and let $\mathfrak{g}_{\mathbb{R}}=\mathfrak{g}^{\sigma^{\prime}}$ be the corresponding almost compact real form. A CSI $\vartheta$ that commutes with $\sigma^{\prime}$ is said to be adapted to $\sigma^{\prime}$ or $\mathfrak{g}_{\mathbb{R}}$. The involution $\sigma=\sigma^{\prime} \vartheta$ (respectively its restriction $\vartheta_{\mathbb{R}}$ to $\mathfrak{g}_{\mathbb{R}}$ or $\sigma_{\mathbb{R}}^{\prime}$ to $\mathfrak{u}=\mathfrak{g}^{\vartheta}$ ) is said to be the Cartan involution of $\sigma^{\prime}$ (respectively of $\mathfrak{g}_{\mathbb{R}}$ or of $\mathfrak{u}$ ). The algebra of fixed points $\mathfrak{k}=\mathfrak{g}_{\mathbb{R}}^{\sigma}=\mathfrak{g}_{\mathbb{R}} \cap \mathfrak{u}=\mathfrak{u}^{\sigma}$ is the maximal compact subalgebra of $\mathfrak{g}_{\mathbb{R}}$. Then $\mathfrak{g}_{\mathbb{R}}=\mathfrak{k} \oplus \mathfrak{p}$ and $\mathfrak{u}=\mathfrak{k} \oplus i \mathfrak{p}$ is a Cartan decomposition of $\mathfrak{g}_{\mathbb{R}}$ and $\mathfrak{u}$ into eigenspaces of $\sigma$. A Cartan subalgebra $\mathfrak{h}$ of $\mathfrak{g}$ is said to be maximally compact for $\sigma^{\prime}\left(\right.$ or $\left.\mathfrak{g}_{\mathbb{R}}\right)$ if it is stable under $\sigma^{\prime}$ and if $-\sigma^{\prime}$ stabilizes a base of $\triangle(\mathfrak{g}, \mathfrak{h})$. 
Proposition 3.6 [Rousseau 1989, Proposition 2.8]. Let $\sigma^{\prime}$ be an SI2 of $\mathfrak{g}$ and let $\mathfrak{g}_{\mathbb{R}}=\mathfrak{g}^{\sigma^{\prime}}$.

(i) There exists a CSI $\vartheta$ adapted to $\sigma^{\prime}$ and a maximally compact CSA $\mathfrak{h}$ for $\sigma^{\prime}$.

(ii) For all maximally compact CSAs $\mathfrak{h}$ for $\sigma^{\prime}$, there exists a CSI $\vartheta$ adapted to $\sigma^{\prime}$ that stabilizes $\mathfrak{h}$, and it is unique up to an interior automorphism fixing $\mathfrak{h}$ and commuting with $\sigma^{\prime}$.

(iii) For all CSIs $\vartheta$ adapted to $\sigma^{\prime}$, there exists a maximally compact Cartan subalgebra for $\sigma^{\prime}$ stabilized by $\vartheta$, and it is unique up to an interior automorphism commuting with $\sigma^{\prime}$ and $\vartheta$.

Proposition 3.7 [Rousseau 1989, Proposition 2.9]. Consider

(i) $\sigma$, an involution of $\mathfrak{g}$ of first type;

(ii) the pairs $\left(\sigma^{\prime}, \mathfrak{h}\right)$ formed of a semiinvolution $\sigma^{\prime}$ of second type, which is not Cartan, and a maximally compact Cartan subalgebra for $\sigma^{\prime}$; and

(iii) the relation $\left(\sigma^{\prime}, \mathfrak{h}\right) \sim \sigma$ if and only if $\sigma$ commutes with $\sigma^{\prime}$, stabilizes $\mathfrak{h}$ and is such that $\sigma \sigma^{\prime}$ is a Cartan semiinvolution of $\mathfrak{g}$.

This relation induces a bijection between the conjugacy classes (under $\operatorname{Int}_{\operatorname{Tr}}(\mathfrak{g})$ or $\operatorname{Aut}(\mathfrak{g}))$ of the involutions of first type (that is, the $\sigma)$ and the pairs $\left(\sigma^{\prime}, \mathfrak{h}\right)$.

Definition. With notations as above, if $\left(\sigma^{\prime}, \mathfrak{h}\right) \sim \sigma$, then we say that $\sigma$ is adapted to $\sigma^{\prime}$, or $\mathfrak{g}_{\mathbb{R}}=\mathfrak{g}^{\sigma^{\prime}}$.

Notation. In what follows, $\dot{\mathfrak{s}}$ will denote a finite-dimensional semisimple Lie algebra over $\mathbb{C}$.

3.8. Adapted Realization of $\mathfrak{g}$. Recall the following definitions from [Ben Messaoud and Rousseau 2003]

Definition. Let $\sigma$ be a finite order automorphism of $\mathfrak{g}$ of first type and $\mathfrak{h}$ a maximally fixed Cartan subalgebra for $\sigma$. A realization of $\mathfrak{g}$ on which $\sigma$ preserves the $\mathbb{Z}$-gradation and for which $\mathfrak{h}$ is the standard Cartan subalgebra is said to be almost adapted to $(\sigma, \mathfrak{h})$. An almost adapted realization $\mathfrak{l}\left(\dot{\mathfrak{s}}, \zeta, \varepsilon_{m}\right)$ for $(\sigma, \mathfrak{h})$ on which $\sigma$ commutes with the translation map $\mathscr{T}: x \rightarrow x \otimes t^{m}$ is said to be adapted to $\sigma$ (of to $(\sigma, \mathfrak{h}))$.

Definition. Let $\sigma^{\prime}$ be a finite order automorphism of $\mathfrak{g}$ of second type, $\mathfrak{h}$ a maximally compact Cartan subalgebra for $\sigma^{\prime}$, and $\sigma$ an involution of first kind associated to the pair $\left(\sigma^{\prime}, \mathfrak{h}\right)$. A realization $\mathfrak{l}\left(\dot{\mathfrak{s}}, \mu, \varepsilon_{\mathfrak{m}}\right)$ of $\mathfrak{g}$ that is adapted to $(\sigma, \mathfrak{h})$ is said to be adapted to $\left(\sigma^{\prime}, \sigma, \mathfrak{h}\right)$ if there exists an involution $\dot{\sigma}$ and a semiinvolution $\dot{\sigma}^{\prime}$ of $\dot{\mathfrak{s}}$ commuting with $\mu$ such that $\dot{\sigma} \dot{\sigma}^{\prime}$ is a Cartan semiinvolution of $\dot{\mathfrak{s}} ; \sigma=\dot{\sigma} \otimes 1$ and $\sigma^{\prime}=\dot{\sigma}^{\prime} \otimes \iota^{\prime}$ on the realization $l^{\prime \prime}\left(\dot{\mathfrak{s}}, \mu, \varepsilon_{m}\right)$; and finally $\sigma^{\prime}(c)=-c$ and $\sigma^{\prime}(d)=-d$. 
It is known from [Ben Messaoud and Rousseau 2003, Proposition 3.4, Theorem 3.5 and Proposition 3.9] that given a finite order automorphism $\sigma$ of first type (respectively $\sigma^{\prime}$ of second type) of $\mathfrak{g}$, there exist realizations of $\mathfrak{g}$ adapted to $\sigma$ (respectively adapted to $\sigma^{\prime}$ ).

3.9. Let $\sigma$ be an involution adapted to a semiinvolution of second type $\sigma^{\prime}$. Since $\sigma$ is an involution of $\mathfrak{g}$ of the first kind, we know by [Bausch 1989, Chapter II] that, up to an interior automorphism, either $\sigma=\rho H$ or $\sigma=H$, where $\rho$ is a diagram automorphism of $\mathfrak{g}=\mathfrak{l}\left(\dot{\mathfrak{g}}, \mu, \varepsilon_{k}\right)$ and $H$ is an interior automorphism of $\mathfrak{g}$ of the form $\exp i \pi \operatorname{ad}\left(h_{0}\right)$, with $h_{0} \in \mathfrak{h}_{\mathbb{Z}}^{\rho}$ where $\mathfrak{h}_{\mathbb{Z}}^{\rho}=\left\{x \in \mathfrak{h}^{\rho} \mid \alpha(x) \in \mathbb{Z}\right.$, for all $\left.\alpha \in \Delta\right\}$. Further $h \in \mathfrak{h}_{\mathbb{Z}}$ can be written as $h=h^{\prime}+\eta d$, with $h^{\prime} \in(\dot{\mathfrak{g}})^{\mu}$ and $\eta \in \mathbb{Z}$.

Proposition 3.10 [Ben Messaoud and Rousseau 2003, Proposition 2.12]. With the notations above, we have the following:

(i) If $\eta$ is even or $k=2$ (that is, $\mathfrak{g}$ is of type Aff 2 ), then the interior involution $H$ respects the $\mathbb{Z}$-gradation of $\mathfrak{g}=l\left(\dot{\mathfrak{g}}, \mu, \varepsilon_{k}\right)$ and commutes with the map $\mathscr{T}: x \rightarrow x \otimes t^{k}$ on $\mathfrak{g}^{\prime \prime}$, and there exists an interior involution $\dot{H}$ of $\dot{\mathfrak{g}}$ commuting with $\mu$ such that $H=\left(\mu^{\eta} \dot{H}\right) \otimes 1$ on $l^{\prime \prime}\left(\dot{\mathfrak{g}}, \mu, \varepsilon_{k}\right)=\mathfrak{g}^{\prime \prime}$.

(ii) If $\eta$ is odd and $k \neq 2$, the interior involution $H$ induces on $\mathfrak{g}^{\prime \prime}$ the automorphism $t^{k} \mapsto-t^{k}$, and $H$ in this case acts on the adapted realization $\mathfrak{l}\left(\dot{\mathfrak{g}} \times \dot{\mathfrak{g}}, \zeta, \varepsilon_{2 k}\right)$ of $\mathfrak{g}$, with $\zeta(x, y)=(y, \mu(x))$.

(iii) Every diagram automorphism of a twisted affine Kac-Moody Lie algebra $\mathfrak{g}=\mathfrak{l}\left(\dot{\mathfrak{g}}, \mu, \varepsilon_{m}\right)$ is $\mathbb{A}$-linear, where $\mathbb{A}$ is the algebra generated by the translation maps $\mathscr{T}^{ \pm}: x \rightarrow x \otimes t^{ \pm m}$.

The following are some examples of realizations of $\mathfrak{g}=\mathfrak{l}\left(\dot{\mathfrak{g}}, \mu, \varepsilon_{k}\right)$ adapted to $\left(\sigma, \sigma^{\prime}, \mathfrak{h}\right)$ where $k=2,3$.

Example 3.11. Let $\mathfrak{g}=\mathfrak{l}(\dot{\mathfrak{g}}, \mu,-1)$, where $\dot{\mathfrak{g}}$ is of type $A_{2 l-1}$.

(i) Let $\sigma=H$, where $H$ is an interior automorphism of $\mathfrak{g}$. Then $\mathfrak{l}(\dot{\mathfrak{g}}, \mu,-1)$ is adapted to $\left(\sigma, \sigma^{\prime}, \mathfrak{h}\right)$ by Proposition 3.10(i).

(ii) Let $\sigma=\rho$, where $\rho$ is a diagram automorphism of $A_{2 l-1}^{(2)}$ such that $\rho\left(\alpha_{0}\right)=\alpha_{1}$, $\rho\left(\alpha_{1}\right)=\alpha_{0}$ and $\rho\left(\alpha_{i}\right)=\alpha_{i}$ for $i \in[2, l]$. In the given realization, we see clearly since $\sigma\left(E_{0} \otimes t\right)=\rho\left(E_{0} \otimes t\right)=e_{1} \otimes 1$ that $\sigma$ cannot be written in the form $\dot{\sigma} \otimes 1$ for any $\dot{\sigma} \in \operatorname{Aut}(\dot{\mathfrak{g}})$. Hence using [Ben Messaoud and Rousseau 2003, 1.7] with $H_{1}=$ $\exp i \pi / 2 \operatorname{ad}\left(\stackrel{\circ}{1}_{1}\right)$, we consider the realization $\mathfrak{l}\left(\dot{\mathfrak{g}}, \mu H_{1}, \varepsilon_{4}\right)$ of $\mathfrak{g}$ that is isomorphic to $\mathfrak{l}(\dot{\mathfrak{g}}, \mu,-1)$ via the map $\psi: \mathfrak{l}(\dot{\mathfrak{g}}, \mu,-1) \rightarrow \mathfrak{l}\left(\dot{\mathfrak{g}}, \mu H_{1}, \varepsilon_{4}\right)$ defined as follows:

$$
\begin{array}{rlrl}
\psi\left(x \otimes t^{j}\right) & =x \otimes t^{2 j+N}+\delta_{j, 0}\left(\stackrel{\circ}{p}_{1}, x\right) C & & \text { for } x \in \dot{\mathfrak{g}}_{j} \text { such that }\left[\stackrel{\circ}{p}_{1}, x\right]=N x, \\
\psi(c) & =2 C, & \psi(d)=\left(D-\stackrel{\circ}{p}_{1}\right) / 2,
\end{array}
$$


where $C$ and $D$ denote the central and gradation elements of $\mathfrak{l}\left(\dot{\mathfrak{g}}, \mu H_{1}, \varepsilon_{4}\right)$, respectively. Since $\left[\stackrel{\circ}{p}_{1}, E_{0}\right]=-E_{0}$ and $\left[\stackrel{\circ}{p}_{1}, e_{1}\right]=e_{1}$, it is easy to see that, under the new realization, $e_{0}=E_{0} \otimes t$ is mapped to $E_{0} \otimes t$ and $e_{1}=e_{1} \otimes 1$ is mapped to $e_{1} \otimes t$, and hence $\rho$ can be written in the form $\dot{\sigma} \otimes 1$, where $\dot{\sigma} \in \operatorname{Aut}(\dot{\mathfrak{g}})$ is an involution such that $\dot{\sigma}\left(E_{0}\right)=e_{1}$ and $\dot{\sigma}\left(e_{i}\right)=e_{i}$ for $i \in[2, l]$. Taking $\dot{\sigma}^{\prime}=\dot{\sigma} \dot{\omega}$, it can be easily seen that $\dot{\sigma} \mu H_{1}=\mu H_{1} \dot{\sigma}$ on $\dot{\mathfrak{g}}$. Hence the realization $\mathfrak{l}\left(\dot{\mathfrak{g}}, \mu H_{1}, \varepsilon_{4}\right)$ is adapted to $\left(\rho, \rho \omega^{\prime}, \mathfrak{h}\right)$.

Example 3.12. Let $\mathfrak{g}=\mathfrak{l}\left(\dot{\mathfrak{g}}, \mu, \varepsilon_{3}\right)$, where $\dot{\mathfrak{g}}$ is of type $D_{4}$.

(i) Let $\sigma=\exp i \pi \operatorname{ad}\left(p_{1}\right)$ or $\exp i \pi \operatorname{ad}\left(\stackrel{\circ}{p}_{2}\right)$. Then by Proposition 3.10(i), ll $\left(\dot{\mathfrak{g}}, \mu, \varepsilon_{3}\right)$ is adapted to $\left(\sigma, \sigma^{\prime}, \mathfrak{h}\right)$.

(ii) Let $\sigma=\exp i \pi \operatorname{ad}\left(p_{0}\right)$. Then by Proposition 3.10(ii), $\mathfrak{l}\left(\dot{\mathfrak{g}} \times \dot{\mathfrak{g}}, \zeta, \varepsilon_{6}\right)$ is an adapted realization of $\mathfrak{l}\left(\dot{\mathfrak{g}}, \mu, \varepsilon_{3}\right)$. It is known from [Ben Messaoud and Rousseau 2003] that for $\zeta \in \operatorname{Aut}(\dot{\mathfrak{g}} \times \dot{\mathfrak{g}})$ as defined above, $\phi_{2}: \mathfrak{l}\left(\dot{\mathfrak{g}}, \mu, \varepsilon_{3}\right) \rightarrow \mathfrak{l}\left(\dot{\mathfrak{g}} \times \dot{\mathfrak{g}}, \zeta, \varepsilon_{6}\right)$, as defined by

$$
\phi_{2}\left(x \otimes t^{j}\right)=\left(x, \varepsilon_{6} x\right) \otimes t^{j}, \quad \phi_{2}(c)=2 c_{2}, \quad \phi_{2}(d)=d_{2},
$$

gives an isomorphism between the two realizations of $\mathfrak{g}$. Here $c_{2}$ and $d_{2}$ denote the central and gradation elements of $\mathfrak{l}\left(\dot{\mathfrak{g}} \times \dot{\mathfrak{g}}, \zeta, \varepsilon_{6}\right)$, respectively.

3.13. Let $\sigma^{\prime}$ be a semiinvolution of second type of $\mathfrak{g}$, and let $\mathfrak{g}_{\mathbb{R}}=(\mathfrak{g})^{\sigma^{\prime}}$ be the corresponding almost compact real form of $\mathfrak{g}$. Let $\omega^{\prime}$ is a Cartan semiinvolution of $\mathfrak{g}$ adapted to $\sigma^{\prime}$. Then $B_{\omega^{\prime}}$ defined by $B_{\omega^{\prime}}(X, Y)=-B\left(X, \omega^{\prime}(Y)\right)$ is a positive definite hermitian form on $\mathfrak{g}_{\mathbb{R}}$, and given $X, Y, Z \in \mathfrak{g}_{\mathbb{R}}$, we have

$$
\begin{aligned}
B_{\omega^{\prime}}(\operatorname{ad} \sigma X(Y), Z) & =-B\left([\sigma X, Y], \omega^{\prime} Z\right) \\
& =B\left(Y,\left[\sigma X, \omega^{\prime} Z\right]\right) \\
& =B\left(Y, \omega^{\prime}\left[\sigma^{\prime} X, Z\right]\right)=-B_{\omega^{\prime}}\left(Y, \operatorname{ad} \sigma^{\prime} X(Z)\right) .
\end{aligned}
$$

Since $\sigma^{\prime} X=X$ for all $X \in \mathfrak{g}_{\mathbb{R}}=\mathfrak{g}^{\sigma^{\prime}}$, we have, with respect to $B_{\omega^{\prime}}$, that

$$
(\operatorname{ad} \sigma X)^{*}=-\operatorname{ad} \sigma^{\prime} X=-\operatorname{ad} X \quad \text { for all } X \in \mathfrak{g}_{\mathbb{R}}=\mathfrak{g}^{\sigma^{\prime}} .
$$

This implies that $\operatorname{ad} X$ is skew-symmetric with purely imaginary eigenvalues for $X \in \mathfrak{k}=\mathfrak{g}^{\sigma}$, and ad $X$ is symmetric with real eigenvalues for $X \in \mathfrak{p}$.

Let $\mathfrak{l}\left(\dot{\mathfrak{s}}, \mu, \varepsilon_{m}\right)$ be a realization of $\mathfrak{g}$ adapted to $\left(\sigma^{\prime}, \sigma, \mathfrak{h}\right)$, where $\dot{\mathfrak{s}}$ is a complex semisimple Lie algebra. Let $\dot{\sigma}, \dot{\sigma}^{\prime} \in \operatorname{Aut}(\dot{\mathfrak{s}})$ such that $\sigma=\dot{\sigma} \otimes 1$ and $\sigma^{\prime}=\dot{\sigma}^{\prime} \otimes \iota^{\prime}$. Let $\dot{\mathfrak{s}}_{\mathbb{R}}=\dot{\mathfrak{s}}^{\dot{\sigma}^{\prime}}$, and let $\dot{\mathfrak{s}}_{\mathbb{R}}=\dot{\mathfrak{k}} \oplus \dot{\mathfrak{p}}$ be its Cartan decomposition with respect to $\dot{\sigma}$.

Lemma 3.14. With the notations above, let $\mathfrak{l}\left(\dot{\mathfrak{s}}, \mu, \varepsilon_{m}\right)$ be a realization of $\mathfrak{g}$ adapted to $\left(\sigma^{\prime}, \sigma, \mathfrak{h}\right)$, and let $\dot{\mathfrak{t}}$ be a $\mu$-stable maximal abelian subspace of $\dot{\mathfrak{k}}=\left(\dot{\mathfrak{s}}_{\mathbb{R}}\right)^{\dot{\sigma}}$. Then $Z_{\dot{\mathfrak{s}}_{\mathbb{R}}}(\dot{\mathfrak{t}})$ is a $\dot{\sigma}$ stable subalgebra of $\dot{\mathfrak{s}}_{\mathbb{R}}$ of the form $Z_{\dot{\mathfrak{s}}_{\mathbb{R}}}(\dot{\mathfrak{t}})=\dot{\mathfrak{t}} \oplus \dot{\mathfrak{a}}$, where $\dot{\mathfrak{a}} \subset \dot{\mathfrak{p}}$ and $\mathfrak{h}_{\mathbb{R}}^{\prime \prime}=(\dot{\mathfrak{t}})^{\mu} \otimes 1 \oplus(\dot{\mathfrak{a}})^{\mu} \otimes 1$ is a Cartan subalgebra of $\mathfrak{g}_{\mathbb{R}}^{\prime \prime}$. 
Proof. Given a finite-dimensional semisimple Lie algebra $\dot{\mathfrak{s}}$ over $\mathbb{C}$, by [Knapp 1996a, Proposition 6.60], $Z_{\dot{\mathfrak{s}}_{\mathbb{R}}}(\dot{\mathfrak{t}})$ is a $\dot{\sigma}$-stable Cartan subalgebra of $\dot{\mathfrak{s}}_{\mathbb{R}}$ of the form $Z_{\dot{\mathfrak{s}}_{\mathbb{R}}}(\dot{\mathfrak{t}})=\dot{\mathfrak{t}} \oplus \dot{\mathfrak{a}}$, with $\dot{\mathfrak{a}} \subset \dot{\mathfrak{p}}$. Since the complex Lie algebra $\mathscr{L}=\left(Z_{\dot{\mathfrak{s}}_{\mathbb{R}}}(\dot{\mathfrak{t}})\right)_{\mathbb{C}}$ is a Cartan subalgebra of $\dot{\mathfrak{s}}$, it is a $\mu$-stable reductive subalgebra of $\dot{\mathfrak{s}}$. Consider the infinite abelian subalgebra $\mathfrak{z}^{\prime \prime}=\mathfrak{l}^{\prime \prime}\left(\mathscr{L}, \mu, \varepsilon_{m}\right)$ of $\mathfrak{g}^{\prime \prime}$. If $x \in \mathscr{L} \subset \dot{\mathfrak{s}}$, then

$$
\sigma\left(x \otimes t^{s}\right)=(\dot{\sigma} \otimes 1)\left(x \otimes t^{s}\right)=\dot{\sigma}(x) \otimes t^{s} .
$$

As $Z_{\dot{\mathfrak{S}}_{\mathbb{R}}}(\dot{\mathfrak{t}})$ is stable under the linear involution $\dot{\sigma}$, we have $\dot{\sigma}(x) \in \mathscr{L}$ for $x \in \mathscr{L}$. Hence $\mathfrak{z}^{\prime \prime}$ is $\sigma$-stable and consequently

$$
\mathfrak{z}^{\prime \prime}=\left(\mathfrak{z}^{\prime \prime} \cap \mathfrak{l}^{\prime \prime}\left(\dot{\mathfrak{p}}_{\mathbb{C}}, \mu, \varepsilon_{m}\right)\right) \oplus\left(\mathfrak{z}^{\prime \prime} \cap \mathfrak{l}^{\prime \prime}\left(\dot{\mathfrak{k}}_{\mathbb{C}}, \mu, \varepsilon_{m}\right)\right) .
$$

But by [Ben Messaoud and Rousseau 2003, Proposition 5.1], the semisimple elements of $\mathfrak{z}^{\prime \prime} \cap \mathfrak{l}^{\prime \prime}\left(\dot{\mathfrak{p}}_{\mathbb{C}}, \mu, \varepsilon_{m}\right)$ are contained in $(\dot{\mathfrak{a}})^{\mu} \otimes 1$ and the semisimple elements of $\mathfrak{z}^{\prime \prime} \cap \mathfrak{l}^{\prime \prime}\left(\dot{\mathfrak{k}}_{\mathbb{C}}, \mu, \varepsilon_{m}\right)$ are contained in $(\dot{\mathfrak{t}})^{\mu} \otimes 1$. Hence an element $x \in \mathfrak{z}^{\prime \prime} \cap \mathfrak{g}_{\mathbb{R}}^{\prime \prime}$ is semisimple if and only if $x \in(\dot{\mathfrak{t}})^{\mu} \otimes 1 \oplus(\dot{\mathfrak{a}})^{\mu} \otimes 1$. Thus $(\dot{\mathfrak{t}})^{\mu} \otimes 1 \oplus(\dot{\mathfrak{a}})^{\mu} \otimes 1$ is a semisimple abelian subalgebra of $\mathfrak{g}_{\mathbb{R}}^{\prime \prime}$. The lemma will now follow if we prove that $\left((\dot{\mathfrak{t}})^{\mu} \otimes 1 \oplus(\dot{\mathfrak{a}})^{\mu} \otimes 1\right)_{\mathbb{C}}=\left((\dot{\mathfrak{t}} \oplus \dot{\mathfrak{a}})^{\mu} \otimes 1\right)_{\mathbb{C}}$ is a Cartan subalgebra of $\mathfrak{g}^{\prime \prime}$

From the definition of the action of $\mu$ on the Lie algebra $l(\dot{\mathfrak{s}}, \mathrm{Id}, 1)^{\prime \prime}$ it clearly follows that

$$
\left((\dot{\mathfrak{t}} \oplus \dot{\mathfrak{a}})^{\mu} \otimes 1\right)_{\mathbb{C}}=(\dot{\mathfrak{t}} \oplus \dot{\mathfrak{a}})^{\mu} \otimes \mathbb{C}=((\dot{\mathfrak{t}} \oplus \dot{\mathfrak{a}}) \otimes \mathbb{C})^{\mu}=\left(Z_{\dot{\mathfrak{s}}_{\mathbb{R}}}(\dot{\mathfrak{t}}) \otimes \mathbb{C}\right)^{\mu}=\mathscr{L}^{\mu}
$$

But by [Knapp 1996a, Proposition 6.60], $\mathscr{Z}$ is a Cartan subalgebra of $\dot{\mathfrak{s}}$, and it is known from [Kac 1990, Chapter 8] that $\mathscr{L}^{\mu}$ is a Cartan subalgebra of $\mathfrak{g}^{\prime \prime}=$ $\mathfrak{l}\left(\dot{\mathfrak{s}}, \mu, \epsilon_{m}\right)^{\prime \prime}$ whenever $\mathscr{Z}$ is a Cartan subalgebra of $\dot{\mathfrak{s}}$. Hence the claim.

3.15. Let $\mathfrak{g}_{\mathbb{R}}$ be an almost compact noncompact real form of $\mathfrak{g}$ corresponding to the SI $2 \sigma^{\prime}$, let $\mathfrak{l}\left(\dot{\mathfrak{s}}, \mu, \varepsilon_{m}\right)$ be a realization of $\mathfrak{g}$ adapted to $\sigma^{\prime}$, let $\sigma$ be a Cartan involution associated to $\sigma^{\prime}$, and let $\mathfrak{g}_{\mathbb{R}}=\mathfrak{k} \oplus \mathfrak{p}$ be the Cartan decomposition of $\mathfrak{g}_{\mathbb{R}}$ with respect to $\sigma$. Let $\mathfrak{h}_{\mathbb{R}}^{\prime \prime}=(\dot{\mathfrak{t}})^{\mu} \otimes 1 \oplus(\dot{\mathfrak{a}})^{\mu} \otimes 1$, with $(\dot{\mathfrak{t}})^{\mu} \subset \dot{\mathfrak{k}}$, and let $(\dot{\mathfrak{a}})^{\mu} \subset \dot{\mathfrak{p}}$ be a $\sigma$-stable Cartan subalgebra of an almost compact real form $\mathfrak{g}_{\mathbb{R}}^{\prime \prime}=\mathfrak{l}\left(\dot{\mathfrak{s}}_{\mathbb{R}}, \mu, \varepsilon_{m}\right)^{\prime \prime}$. We say $\mathfrak{h}_{\mathbb{R}}^{\prime \prime}$ is maximally compact if $\operatorname{dim}(\dot{\mathfrak{t}})^{\mu}$ is as large as possible. It clearly follows from Section 3.13 and Lemma 3.14 that all the real roots of a maximally compact Cartan subalgebra $\mathfrak{h}_{\mathbb{R}}$ of an almost compact real form $\mathfrak{g}_{\mathbb{R}}$ have real eigenvalues on $(\dot{\mathfrak{a}})^{\mu} \otimes 1$ and imaginary eigenvalues on $(\dot{\mathfrak{t}})^{\mu} \otimes 1$. A real root is called $\sigma$-real if it takes real values on $\mathfrak{h}_{\mathbb{R}}$ (that is, it vanishes on $(\dot{\mathfrak{t}})^{\mu} \otimes 1$ ), $\sigma$-imaginary if it takes purely imaginary values on $\mathfrak{h}_{\mathbb{R}}$ (that is, vanishes on $(\dot{\mathfrak{a}})^{\mu} \otimes 1$ ), and $\sigma$-complex otherwise. For any $\alpha \in \triangle^{\text {re }}$, let $X_{\alpha} \in \mathfrak{g}_{\alpha}$. Then

$$
\left[H, \sigma X_{\alpha}\right]=\sigma\left[\sigma^{-1} H, X_{\alpha}\right]=\alpha\left(\sigma^{-1} H\right) \sigma X_{\alpha} .
$$


Hence $\sigma \alpha(H)=\alpha\left(\sigma^{-1} H\right)$ is a root. If $\alpha$ is $\sigma$-imaginary, then $\sigma \alpha=\alpha$. In this case, $\mathfrak{g}_{\alpha}$ is $\sigma$-stable, and we have $\mathfrak{g}_{\alpha}=\left(\mathfrak{g}_{\alpha} \cap \mathfrak{k}\right) \oplus\left(\mathfrak{g}_{\alpha} \cap \mathfrak{p}\right)$. Since $\mathfrak{g}_{\alpha}$ is 1-dimensional, $\mathfrak{g}_{\alpha} \subset \mathfrak{k}$ or $\mathfrak{g}_{\alpha} \subset \mathfrak{p}$. A $\sigma$-imaginary real root $\alpha$ is said to be compact if $\mathfrak{g}_{\alpha} \subset \mathfrak{k}$, and noncompact if $\mathfrak{g}_{\alpha} \subset \mathfrak{p}$.

3.16. Let $\alpha \in \triangle^{\text {re }}$ be $\sigma$-real. Since $\sigma \alpha=-\alpha, \omega^{\prime}(\alpha)=-\alpha$, and $\sigma \sigma^{\prime}$ is an $\operatorname{Int}(\mathfrak{g})$-conjugate of $\omega^{\prime}$, we have $\sigma^{\prime}\left(H_{\alpha}\right)=H_{\alpha}$. Therefore $H_{\alpha} \in \mathfrak{p} \subset \mathfrak{g}_{\mathbb{R}}$ is $\operatorname{ad}_{\mathfrak{g}}$ diagonalizable with real eigenvalues. For $X_{ \pm \alpha} \in \mathfrak{g}_{ \pm \alpha}$ and $H \in \mathfrak{h}^{\sigma^{\prime}}$, we have

$$
\left[H, \sigma^{\prime} X_{ \pm \alpha}\right]=\sigma^{\prime}\left[\sigma^{\prime}(H), X_{ \pm \alpha}\right]= \pm \alpha\left(\sigma^{\prime}(H)\right) \sigma^{\prime} X_{ \pm \alpha}= \pm \alpha(H) \sigma^{\prime} X_{ \pm \alpha} .
$$

Therefore $\sigma^{\prime}\left(\mathfrak{g}_{ \pm \alpha}\right) \subset \mathfrak{g}_{ \pm \alpha}$. Fixing $E_{\alpha} \in \mathfrak{g}_{\alpha}$ and $E_{-\alpha} \in \mathfrak{g}_{-\alpha}$ so that $B\left(E_{\alpha}, E_{-\alpha}\right)=1$, we get $\left[E_{\alpha}, E_{-\alpha}\right]=H_{\alpha}$. Since $\operatorname{dim} \mathfrak{g}_{ \pm \alpha}=1$ for $\alpha \in \triangle^{\text {re }}$, the fact that $\sigma^{\prime}$ is an involution implies that $\sigma^{\prime}\left(E_{ \pm \alpha}\right)=E_{ \pm \alpha}$ or $\sigma^{\prime}\left(E_{ \pm \alpha}\right)=-E_{ \pm \alpha}$.

Case 1: If $\sigma^{\prime}\left(E_{ \pm \alpha}\right)=E_{ \pm \alpha}$, then $\mathbb{R} E_{\alpha} \oplus \mathbb{R} H_{\alpha} \oplus \mathbb{R} E_{-\alpha} \subset \mathfrak{g}_{\mathbb{R}}$. By suitably scaling $E_{\alpha}$ and $E_{-\alpha}$, we can find $E_{\alpha} \in \mathfrak{g}_{\alpha}, E_{-\alpha} \in \mathfrak{g}_{-\alpha}$ and $H_{\alpha} \in \mathfrak{h}^{\sigma^{\prime}}$ such that

$$
\left[H_{\alpha}, E_{\alpha}\right]=2 E_{\alpha}, \quad\left[H_{\alpha}, E_{-\alpha}\right]=-2 E_{-\alpha}, \quad\left[E_{\alpha}, E_{-\alpha}\right]=H_{\alpha} .
$$

From the definitions of the Cartan semiinvolution and the Cartan involution $\sigma$ adapted to $\sigma^{\prime}$, it now clear that $\sigma\left(E_{\alpha}\right)=-E_{-\alpha}$ and $\sigma\left(E_{-\alpha}\right)=-E_{\alpha}$. Hence $E_{\alpha}-E_{-\alpha} \in \mathfrak{k}$. Corresponding to such a $\sigma$-real root $\alpha$, define an automorphism

$$
D_{\alpha}^{r}=\operatorname{Ad}\left(\exp (\pi / 4)\left(-E_{-\alpha}-E_{\alpha}\right)\right) .
$$

Following calculations similar to those in [Knapp 1996a, Proposition 6.52], we get $D_{\alpha}^{r}\left(H_{\alpha}\right)=\left(E_{\alpha}-E_{-\alpha}\right)$.

Case 2: If $\sigma^{\prime}\left(E_{ \pm \alpha}\right)=-E_{ \pm \alpha}$, then $\mathbb{R} i E_{\alpha} \oplus \mathbb{R} H_{\alpha} \oplus \mathbb{R} i E_{-\alpha} \subset \mathfrak{g}_{\mathbb{R}}$. Then, by suitably scaling $E_{\alpha}$ and $E_{-\alpha}$, we can find $E_{\alpha} \in \mathfrak{g}_{\alpha}, E_{-\alpha} \in \mathfrak{g}_{-\alpha}$ and $H_{\alpha} \in \mathfrak{h}^{\sigma^{\prime}}$ such that

$$
\left[H_{\alpha}, i E_{\alpha}\right]=2 i E_{\alpha}, \quad\left[H_{\alpha}, i E_{-\alpha}\right]=-2 i E_{-\alpha}, \quad\left[i E_{\alpha}, i E_{-\alpha}\right]=-H_{\alpha} .
$$

From the definitions of the Cartan semiinvolution and the Cartan involution $\sigma$ adapted to $\sigma^{\prime}$, it now clear that $\sigma\left(E_{\alpha}\right)=E_{-\alpha}$ and $\sigma\left(E_{-\alpha}\right)=E_{\alpha}$. Consequently $i\left(E_{\alpha}+E_{-\alpha}\right) \in \mathfrak{k}$. Corresponding to such a $\sigma$-real root $\alpha$ define an automorphism

$$
D_{\alpha}^{\mathrm{im}}=\operatorname{Ad}\left(\exp (i \pi / 4)\left(E_{-\alpha}-E_{\alpha}\right)\right) .
$$

Following calculations similar to those in [Knapp 1996a, Proposition 6.52], we get $D_{\alpha}^{\mathrm{im}}\left(H_{\alpha}\right)=i\left(E_{\alpha}+E_{-\alpha}\right)$.

Given a $\sigma$-real root $\alpha, D_{\alpha}^{r}\left(H_{\alpha}\right)$ and $D_{\alpha}^{\mathrm{im}}\left(H_{\alpha}\right)$ are images of semisimple elements and hence semisimple. Hence, it follows from the discussion above that

- if $\sigma^{\prime}$ fixes $\mathfrak{g}_{\alpha} \oplus \mathfrak{g}_{-\alpha}$, then $D_{\alpha}^{r}$ increases the dimension of $(\mathfrak{\mathfrak { t }})^{\mu} \otimes 1$ by 1 ;

- if $-\sigma^{\prime}$ fixes $\mathfrak{g}_{\alpha} \oplus \mathfrak{g}_{-\alpha}$, then $D_{\alpha}^{\mathrm{im}}$ increases the dimension of $(\dot{\mathfrak{t}})^{\mu} \otimes 1$ by 1 . 
Now replacing $d_{\alpha}$ by $D_{\alpha}^{r}$ and $D_{\alpha}^{\mathrm{im}}$ as appropriate, the same proof as [Batra 2000, Proposition 3.8] shows this:

Lemma 3.17. A $\sigma$-stable Cartan subalgebra $\mathfrak{h}_{\mathbb{R}}^{\prime \prime}$ of $\mathfrak{g}_{\mathbb{R}}^{\prime \prime}$ is maximally compact if and only if there are no $\sigma$-real real roots in $\mathfrak{h}_{\mathbb{R}}^{\prime \prime}{ }^{1}$

Remark 3.18. As a consequence, we have $\mathfrak{h}_{\mathbb{R}}=\mathfrak{h} \cap \mathfrak{g}^{\sigma^{\prime}}=\mathfrak{h} \cap \mathfrak{g}_{\mathbb{R}}=i(\mathfrak{h} \cap \mathfrak{k}) \oplus(\mathfrak{h} \cap \mathfrak{p})$,

with

$\mathfrak{h} \cap \mathfrak{p}=\bigoplus_{\beta \in \triangle^{\mathrm{re}}} \mathbb{R}\left(p_{\beta}-p_{\sigma \beta}\right)$,

and

$$
\mathfrak{h} \cap \mathfrak{k}=\left(\bigoplus_{\alpha \in \Delta^{\mathrm{re}}} \mathbb{R} p_{\alpha}\right) \oplus\left(\bigoplus_{\beta \in \Delta^{\mathrm{re}}} \mathbb{R}\left(p_{\beta}+p_{\sigma \beta}\right)\right) \quad \text { (modulo the center), }
$$

where $\left(p_{\gamma}\right)_{\gamma \in \Delta^{\text {re }}} \subset \mathfrak{h}$ is the dual basis of the real roots. Clearly $(\mathfrak{h} \cap \mathfrak{k}) \oplus i(\mathfrak{h} \cap \mathfrak{p})$ is a maximally compact Cartan subalgebra of $\mathfrak{g}^{\varsigma}$, where $\varsigma$ is a compact semiinvolution adapted to $\sigma^{\prime}$ (that is, $\sigma^{\prime}=\varsigma \sigma$ ).

\section{Vogan diagrams}

4.1. Let $\mathfrak{g}_{\mathbb{R}}$ be an almost compact real form of $\mathfrak{g}$, and let $\sigma^{\prime}$ be a semiinvolution of second type associated to $\mathfrak{g}_{\mathbb{B}}$. Let $\sigma$ be a Cartan involution adapted to $\sigma^{\prime}$, and let $\mathfrak{g}_{\mathbb{R}}=\mathfrak{k} \oplus \mathfrak{p}$ be the corresponding Cartan decomposition of $\mathfrak{g}_{\mathbb{R}}$. Let

$$
\mathfrak{h}_{\mathbb{R}}=i\left((\dot{\mathfrak{t}})^{\mu} \otimes 1\right) \oplus(\dot{\mathfrak{a}})^{\mu} \otimes 1 \oplus \mathbb{R} i c \oplus \mathbb{R} i d
$$

be a maximally compact Cartan subalgebra of $\mathfrak{g}_{\mathbb{R}}$. By Lemma 3.17, $\mathfrak{h}_{\mathbb{R}}$ does not have any $\sigma$-real roots. Choose a positive system $\Delta^{+}$for $\triangle(\mathfrak{g}, \mathfrak{h})$, built from a basis of $i\left((\dot{\mathfrak{t}})^{\mu} \otimes 1\right)$ followed by a basis of $(\dot{\mathfrak{a}})^{\mu} \otimes 1$. Since $\left.\sigma\right|_{i(\dot{\mathfrak{t}})^{\mu} \otimes 1}=\mathrm{Id},\left.\sigma\right|_{(\dot{\mathfrak{a}})^{\mu} \otimes 1}=-\mathrm{Id}$ and $\mathfrak{h}_{\mathbb{R}}$ contains no $\sigma$-real roots, $\sigma\left(\Delta^{+}\right)=\Delta^{+}$. Thus $\sigma$ fixes the $\sigma$-imaginary roots and permutes in 2-cycles the $\sigma$-complex roots.

A Vogan diagram of the triple $\left(\mathfrak{g}_{\mathbb{R}}, \mathfrak{h}_{\mathbb{R}}, \Delta^{+}\right)$is a Dynkin diagram of $\Delta^{+}$with the 2-element orbits of $\sigma$ so labeled and with the one element orbit painted or not according to whether the corresponding $\sigma$-imaginary simple root is noncompact or compact. In addition to this, the underlying Dynkin has numerical labels as given in Table 1.

4.2. The Cartan involution $\sigma$ is an involution of first kind. Let the realization $\mathfrak{l}\left(\dot{\mathfrak{s}}, \zeta, \varepsilon_{m}\right)$ of $\mathfrak{g}$ be adapted to $\sigma$. By Section 3.9, $\sigma$ is of the form $\rho^{J} \exp i \pi \operatorname{ad}\left(h_{0}\right)$, for $h_{0} \in \mathfrak{h}_{\mathbb{Z}}^{\rho}$ and $j \in \mathbb{Z}_{2}$, where $\rho$ is a diagram automorphism of $S(A)$. Then the base $\Pi=\left(\alpha_{j}\right)_{j \in[0, l]}$ can be chosen so that either $h_{0}=p_{j} \in \mathfrak{h}$ for some $j \in[0, l]$ or $h_{0}=\stackrel{\circ}{p}_{j} \in \mathfrak{h} \subset \mathfrak{h}$ for some $j \in[1, l]$, where the set $\left(p_{j}\right)_{j \in[0, l]} \subset \mathfrak{h}$ satisfies the property that $\alpha_{k}\left(p_{j}\right)=\delta_{k, j}$ for $k, j \in[0, l]$ (see Section 2.5$)$ and $\left(\stackrel{\circ}{p}_{j}\right)_{j \in[1, l]}$ is a dual

\footnotetext{
${ }^{1}$ An independent proof of Lemma 3.17 is given in [Ben Messaoud and Rousseau 2007, 2.6(iii)].
} 
basis of the base $\left(\alpha_{j}\right)_{j \in[1, l]}$ of $\mathfrak{g} \subset \mathfrak{g}$. Since $\alpha_{k}\left(p_{j}\right)=\delta_{k, j}$ for $k, j \in\{0,1, \ldots, l\}$, it can be easily seen that

$$
\exp i \pi \operatorname{ad}\left(p_{k}\right)\left(e_{j}\right)=(-1)^{\delta_{k, j}} e_{k} \quad \text { for } j \in\{0,1, \ldots, l\} .
$$

If the realization $\mathfrak{l}\left(\dot{\mathfrak{s}}, \zeta, \varepsilon_{m}\right)$ of $\mathfrak{g}$ is adapted to $\sigma=\exp i \pi \operatorname{ad}\left(p_{0}\right)$, then it follows from the definition of adapted realization and the discussion following Proposition 3.10 that

$$
\begin{aligned}
& \text { for } m=2, \quad \dot{\mathfrak{s}}=\dot{\mathfrak{g}}, \quad \zeta \otimes 1\left(e_{0}\right)=-e_{0}, \quad \zeta \otimes 1\left(e_{j}\right)=e_{j} \quad \text { for } j \neq 0 ; \\
& \text { for } m=6, \quad \dot{\mathfrak{s}}=\dot{\mathfrak{g}} \times \dot{\mathfrak{g}}, \quad \zeta^{3} \otimes 1\left(e_{0}\right)=-e_{0}, \quad \zeta \otimes 1\left(e_{j}\right)=e_{j} \quad \text { for } j \neq 0 .
\end{aligned}
$$

By Lemma 3.14, $\dot{\mathfrak{h}}^{\zeta}$ is the Cartan subalgebra of $\mathfrak{l}\left(\dot{\mathfrak{s}}, \zeta, \varepsilon_{m}\right)^{\prime \prime}$ and exp $\left.i \pi \operatorname{ad}\left(p_{0}\right)\right|_{\mathfrak{h}^{\zeta}}=$ $\operatorname{Id}=\left.(\zeta \otimes 1)\right|_{\mathfrak{h}^{\zeta}}$. Since $\left\{e_{j}, f_{j}\right\}_{j \in[0, l]}$ and $\dot{\mathfrak{h}}^{\zeta}$ generate $\mathfrak{g}$, we get

$$
\exp i \pi \operatorname{ad}\left(p_{0}\right)= \begin{cases}\zeta \otimes 1 & \text { for } m=2 \\ \zeta^{3} \otimes 1 & \text { for } m=6 .\end{cases}
$$

Also $\alpha_{k}\left(\stackrel{\circ}{p}_{j}\right)=\delta_{k, j}$ for $k, j \in\{1, \ldots, l\}$, and $\alpha_{0}\left(\stackrel{\circ}{p}_{j}\right)=-a_{j}$ for $j \in\{1, \ldots, l\}$. Since $p_{0}=d$ and $p_{j}=\stackrel{\circ}{p}_{j}+a_{j} d$ for $j \in[1, l]$, using (4-1) we get

$$
\begin{array}{ll}
\exp i \pi \operatorname{ad}\left(\dot{\circ}_{k}\right)\left(e_{j}\right)=(-1)^{\delta_{k, j}} e_{k} & \text { for } k, j \in\{1, \ldots, l\}, \\
\exp i \pi \operatorname{ad}\left(\stackrel{\circ}{p}_{k}\right)\left(e_{0}\right)=(-1)^{a_{k}} e_{0} & \text { for } k \in\{1, \ldots, l\},
\end{array}
$$

Thus it follows from (4-1) and (4-2) that for $k \in\{1,2, \ldots, l\}$,

$$
\exp i \pi \operatorname{ad}\left(p_{k}\right)= \begin{cases}\exp i \pi \operatorname{ad}\left(\dot{p}_{k}\right) & \text { if } a_{k} \text { is even } \\ \left(\exp i \pi \operatorname{ad}\left(\stackrel{\circ}{p}_{k}\right) \zeta\right) \otimes 1 & \text { if } a_{k} \text { is odd. }\end{cases}
$$

From the symmetry of the diagram, it is clear that for $\mathfrak{g}=\mathfrak{l}\left(\dot{\mathfrak{g}}, \mu, \varepsilon_{k}\right)$, the involution of first type $\sigma$ is of the form $\rho \exp i \pi \operatorname{ad}\left(p_{j}\right)$, where $j \in[1, l]$, only when $k=2$. In this case $\mathfrak{l}\left(\dot{\mathfrak{g}}, \zeta, \varepsilon_{2 k}\right)=\mathfrak{l}\left(\dot{\mathfrak{g}}, \mu \exp (i \pi / 2) \operatorname{ad}\left(\stackrel{\circ}{p}_{\rho_{(0)}}\right), \varepsilon_{2 k}\right)$ is an adapted realization of $\mathfrak{g}$, where ${\stackrel{\circ}{\rho_{(0)}}}_{\rho^{\prime}}$ is the dual of $\rho\left(\alpha_{0}\right)$ in $(\dot{\mathfrak{h}})^{\mu}$, and one gets that, for $j \in\{1, \ldots, l\}$,

$$
\exp i \pi \operatorname{ad}\left(p_{j}\right)= \begin{cases}\exp i \pi \operatorname{ad}\left(\stackrel{\circ}{p}_{j}\right) & \text { if } a_{j} \text { is even, } \\ \left(\exp i \pi \operatorname{ad}\left(\stackrel{\circ}{p}_{j}\right) \zeta^{2}\right) \otimes 1 & \text { if } a_{j} \text { is odd. }\end{cases}
$$

4.3. It follows from (4-1) that the Vogan diagram associated to the involution $\exp i \pi \operatorname{ad}\left(p_{j}\right)$ for $j \in[0, l]$ has exactly one painted vertex, namely the $j$-th vertex. By (4-2), the Vogan diagrams associated to the involution $\exp i \pi \operatorname{ad}\left(\stackrel{\circ}{p}_{j}\right)$ for $j \in[1, l]$ have exactly one painted vertex, namely the $j$-th vertex, if $a_{j}$ is even, and exactly two painted vertices, namely the $j$-th and the 0 -th vertices, if $a_{j}$ is odd. Note that $\sigma$ is of the form $\rho H$ for $\rho \neq \operatorname{Id}$ only when $\mathfrak{g}$ is of type $A_{2 l-1}^{(2)}$ or $D_{l+1}^{(2)}$. In both the cases $\alpha_{0}$ is a $\sigma$-complex root, and hence the interior automorphism $H$ is of the form $\exp i \pi \operatorname{ad}\left(p_{j}\right)$ for some $j \in[1, l]$ such that $\rho\left(\alpha_{j}\right)=\alpha_{j}$. 
4.4. Equivalence of Vogan diagrams. [Knapp 1996a, Chapter VI, Exercise 18] defines an operation $R[j]$ on the Vogan diagram of a simple finite-dimensional Lie algebra $\mathfrak{g}$ as follows:

$R[j]$ acts on the base $\stackrel{\pi}{\pi}$ of $\mathfrak{g}$ by the reflection corresponding to the noncompact simple root $\alpha_{j}$. As a consequence of $R[j]$, the color of $\alpha_{j}$ and all vertices not adjacent to $\alpha_{j}$ remain unchanged; if $\alpha_{k}$ is joined to $\alpha_{j}$ by a double edge and $\alpha_{j}$ is the smaller root, then the color of $\alpha_{k}$ remains unchanged, and if $\alpha_{k}$ is joined to $\alpha_{j}$ by single or triple lines, then the color of $\alpha_{k}$ is reversed.

Equivalence 4.5. Generate an equivalence relation among Vogan diagrams by applying

(i) a diagram automorphism of the Dynkin diagram $S(A)$, and

(ii) a sequence of $R[j] \mathrm{s}$ for $j \in\{1,2, \ldots, l\}$ if the $j$-th vertex is colored.

Given a Lie algebra $\mathfrak{g}=\mathfrak{l}\left(\dot{\mathfrak{g}}, \mu, \varepsilon_{k}\right)$, the set of involutions $\left\{\exp i \pi \operatorname{ad}\left(\stackrel{\circ}{p}_{k}\right)\right\}_{k \in[1, l]}$ is a subset of $\operatorname{Aut}\left((\dot{\mathfrak{g}})^{\mu}\right)$. Observe that the base of the twisted affine Kac-Moody Lie algebra $\mathfrak{g}$ is given by $\stackrel{\circ}{\pi} \cup\left\{\alpha_{0}\right\}$, where $\alpha_{0}=\delta-\theta$ for $\mathfrak{g}$ not of type $A_{2 l}^{(2)}$, and $\alpha_{0}=\delta-2 \theta$ for $\mathfrak{g}$ of type $A_{2 l}^{(2)}$, where $\theta \in \triangle_{+}$; also, $\stackrel{\circ}{\pi}$ is a base of $(\dot{\mathfrak{g}})^{\mu}$, and $\stackrel{W}{W} \ltimes T$ is the Weyl group of $\mathfrak{g}$ (see Section 1) with $w(\delta)=\delta$ for $w \in W$. Hence, if the compact real forms of $\mathfrak{g}$ corresponding to the involutions exp $i \pi \operatorname{ad}\left(\stackrel{\circ}{p}_{k}\right)$ and $\exp i \pi \operatorname{ad}\left(\stackrel{\circ}{p}_{j}\right)$ are isomorphic via an isomorphism induced by $\stackrel{\circ}{W}$, then $\stackrel{\circ}{W} \subset W$ induces an isomorphism between the corresponding almost compact real forms of $\mathfrak{g}$. As a consequence we get the second equivalence relation as defined above.

The following are some examples of equivalent Vogan diagrams.

Example 4.6 $D_{l+1}^{(2)}$ for $l \geq 2$. Equivalence 4.5(i) implies
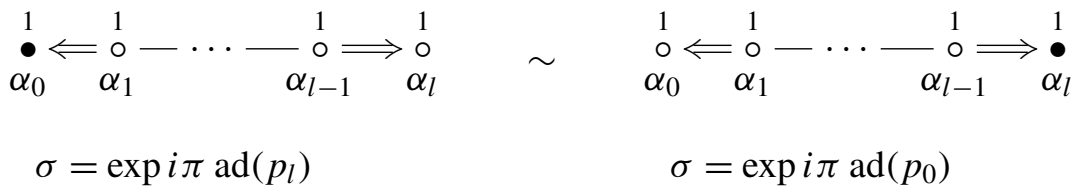

Example 4.7 $A_{2 l-1}^{(2)}$. Equivalence 4.5(ii), with the sequence $R[l-1] \cdots R[2] R[1]$, implies

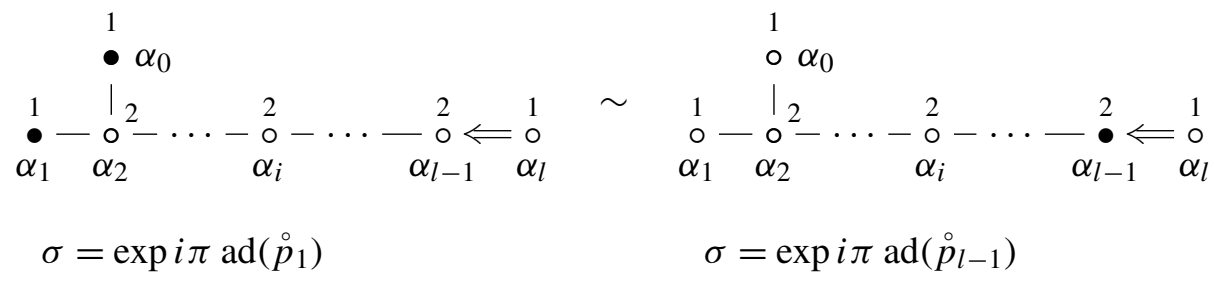


Example 4.8 $A_{2 l-1}^{(2)}$. With the sequence $R[4] R[3] R[2] R[5] R[2] R[4] R[3]$, Equivalence 4.5 (ii) gives

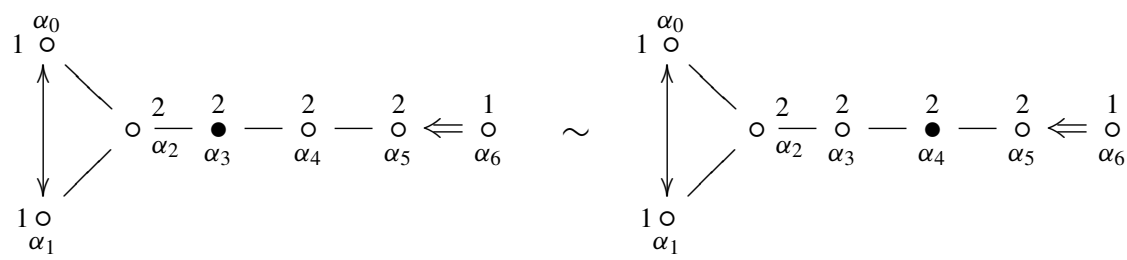

Example 4.9 $A_{2 l-1}^{(2)}$. By Equivalence 4.5(i), we have
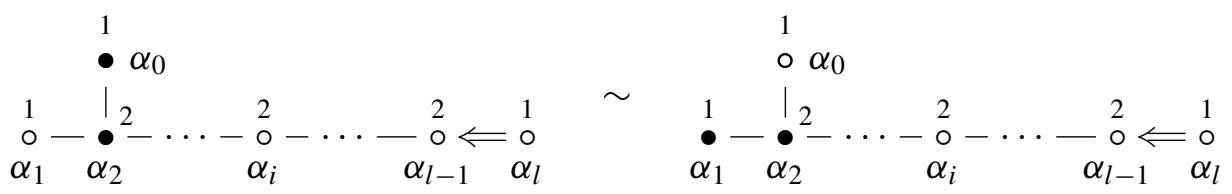

which, by Equivalence 4.5(ii) with the sequence $R[1]$, is in turn equivalent to

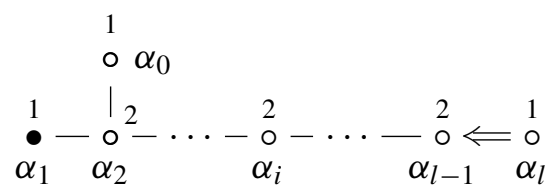

In this example, Equivalence 4.5(ii) gives $r_{1}\left(\alpha_{2}\right)=\alpha_{2}+\alpha_{1}$ and $r_{1}\left(\alpha_{0}\right)=\alpha_{0}$, and hence the final diagram is a consequence of the fact that $[\mathfrak{k}, \mathfrak{k}] \subset \mathfrak{k},[\mathfrak{k}, \mathfrak{p}] \subset \mathfrak{p}$ and $[\mathfrak{p}, \mathfrak{p}] \subset \mathfrak{k}$.

Remark 4.10. As a consequence of Section 4.2 and the equivalence relation between the Vogan diagrams, there exists a base $\Pi^{\prime}$ of a twisted affine Kac-Moody Lie algebra $\mathfrak{g}$ with corresponding positive root system $\triangle^{\prime}$ such that the Vogan diagram associated to $\left(\mathfrak{g}_{\mathbb{R}}, \mathfrak{h}_{\mathbb{R}}, \Delta^{\prime}\right)$ has at most two painted simple roots. Hence every Vogan diagram is equivalent to one with at most two painted simple roots.

Definition. An abstract Vogan diagram is an irreducible abstract Dynkin diagram of a twisted affine Kac-Moody Lie algebra $\mathfrak{g}$ indicated with the following additional structures:

- a diagram automorphism of order 1 or 2 , indicated by labeling the 2 -element orbits;

- a subset of the 1-element orbits, indicated by painting the vertices corresponding to the members of the subset; and

- enumerated vertices, as given in Table 1.

Every Vogan diagram is of course an abstract Vogan diagram. 
Theorem 4.11. Let an abstract Vogan diagram for a twisted affine Kac-Moody Lie algebra be given. Then there exists an almost compact real form of a twisted affine Kac-Moody Lie algebra such that the given diagram is the Vogan diagram of this almost compact real form.

Proof. It is known from [Kac 1990], that a GCM $A$ can be uniquely associated to a Dynkin diagram $S(A)$ and its enumeration of vertices. By [Kac 1990, Proposition 1.1], there exists a unique up-to-isomorphism realization for every GCM $A$. Following [Kac 1990, Section 1.3], one can associate to $A$ a Kac-Moody Lie algebra $\mathfrak{g}=\mathfrak{g}(A)$.

By Proposition 3.7, there exists a one-to-one correspondence between involutions $\sigma$ of first kind and the pairs $\left(\sigma^{\prime}, \mathfrak{h}\right)$ formed of a semiinvolution $\sigma^{\prime}$ of second type and a maximally compact Cartan subalgebra for $\sigma^{\prime}$. Thus if the involution $\sigma$ of $\mathfrak{g}(A)$ of the first kind can be extracted from the additional structural information superimposed on the Vogan diagram, then one can associate to the Vogan diagram an almost compact real form of a twisted affine Kac-Moody Lie algebra. We shall now prescribe an algorithm to associate an involution of the first kind $\sigma$ to a given Vogan diagram.

Let $V(A)$ denote a Vogan diagram of $\mathfrak{g}(A)$. By Remark 4.10, $V(A)$ is equivalent to a Vogan diagram of $\mathfrak{g}(A)$ with at most two painted simple roots.

First, if $V(A)$ has no painted simple roots and no 2-element orbits, then it corresponds the compact form $\mathfrak{u}(A)$ of $\mathfrak{g}(A)$ and $\sigma=\mathrm{Id}$ in this case.

Second, suppose $V(A)$ contains no 2-element orbit. Then, for $j \in[0, l]$,

$\sigma= \begin{cases}\exp i \pi \operatorname{ad}\left(p_{j}\right) & \text { if only the } j \text {-th vertex is painted, } \\ \exp i \pi \operatorname{ad}\left(\dot{p}_{j}\right) & \text { if } a_{j} \text { is odd and the } 0 \text {-th and } j \text {-th vertices are painted. }\end{cases}$

Third, suppose $V(A)$ contains 2-element orbits. If $\rho$ denotes the Dynkin diagram automorphism in $V(A)$ then from Section 4.3 it follows that

$$
\sigma= \begin{cases}\rho \exp i \pi \operatorname{ad}\left(p_{i}\right) & \text { for } i \in[1, l] \text { if only the } i \text {-th vertex is painted, } \\ \rho & \text { if no vertices are painted. }\end{cases}
$$

The association of $\sigma$ with $V(A)$ thus completes the proof of the theorem.

Theorem 4.12. If two almost compact real forms of a twisted affine Kac-Moody Lie algebra $\mathfrak{g}$ have equivalent Vogan diagrams, they are isomorphic.

Remark. Owing to the definition of the equivalence relation between Vogan diagrams, to prove the theorem, it suffices to show that two almost compact real forms having the same Vogan diagram are isomorphic.

Proof. Let $\mathfrak{g}_{1}$ and $\mathfrak{g}_{2}$ be two almost compact real forms of $\mathfrak{g}$ having the same Vogan diagram. As they both have the same Dynkin diagram with the same enumeration on the vertices, the same generalized Cartan matrix $A$ is associated with both $\mathfrak{g}_{1}$ 
and $\mathfrak{g}_{2}$. Thus the unique twisted Kac-Moody Lie algebra $\mathfrak{g}=\mathfrak{g}(A)$ is the complexification of $\mathfrak{g}_{1}$ and $\mathfrak{g}_{2}$. By Proposition 3.6 there exists Cartan semiinvolutions $\vartheta_{1}$ and $\vartheta_{2}$ adapted to $\mathfrak{g}_{1}$ and $\mathfrak{g}_{1}$ respectively. Let $\mathfrak{u}_{j}=\mathfrak{g}^{\vartheta_{j}}$ for $j=1,2$ be the corresponding compact real forms of $\mathfrak{g}$. Let $\sigma$ be the involution represented by the Vogan diagram. Then for $j=1,2,\left.\sigma\right|_{\mathfrak{u}_{j}}=\varpi_{j}$ is the corresponding Cartan involution on $\mathfrak{u}_{j}$. Since by [Rousseau 1989, Theorem 4.6] all Cartan semiinvolutions are conjugate by $\operatorname{Int}(\mathfrak{g})$, there exists an $x \in \operatorname{Int}(\mathfrak{g})$ such that $x \vartheta_{1} x^{-1}=\vartheta_{2}$ and consequently $x \cdot \mathfrak{u}_{1}=\mathfrak{u}_{2}$. Because $x \cdot \mathfrak{g}_{1}$ is isomorphic to $\mathfrak{g}_{1}$, we may assume without loss of generality from the outset that $\mathfrak{u}_{1}=\mathfrak{u}_{2}=\mathfrak{u}$ and we have $\varpi_{j}(\mathfrak{u})=\mathfrak{u}$ for $j=1,2$.

Let $\mathfrak{h}_{1}=\mathfrak{t}_{1} \oplus \mathfrak{a}_{1}$ and $\mathfrak{h}_{2}=\mathfrak{t}_{2} \oplus \mathfrak{a}_{2}$ be the Cartan decompositions of the Cartan subalgebras of $\mathfrak{g}_{1}$ and $\mathfrak{g}_{2}$, respectively, where $\mathfrak{t}_{j}$ and $\mathfrak{a}_{j}$ for $j=1,2$ are respectively the +1 and -1 eigenspaces of $\sigma$ in $\mathfrak{h}_{j}$. Consequently, $\mathfrak{t}_{j} \oplus i \mathfrak{a}_{j}$ for $j=1,2$ is a maximal abelian subspace of $\mathfrak{u}$ and hence by Remark 3.18 is also a maximally compact Cartan subalgebra of $\mathfrak{u}$. Therefore by [Rousseau 1989, Proposition 4.9c], $\mathfrak{t}_{1} \oplus i \mathfrak{a}_{1}$ and $\mathfrak{t}_{2} \oplus i \mathfrak{a}_{2}$ are conjugate by an element $k \in \operatorname{Int}(\mathfrak{u})$. Replacing $\mathfrak{g}_{2}$ by $k \mathfrak{g}_{2}$ and arguing as above, we may assume that $\mathfrak{t}_{1} \oplus i \mathfrak{a}_{1}=\mathfrak{t}_{2} \oplus i \mathfrak{a}_{2}$. Thus $\mathfrak{t}_{1} \oplus i \mathfrak{a}_{1}$ and $\mathfrak{t}_{2} \oplus i \mathfrak{a}_{2}$ have the same complexification, which is denoted by $\mathfrak{h}$.

Now the complexifications of $\mathfrak{g}$ and $\mathfrak{h}$ have been aligned, and the root systems are the same. Let the respective positive root systems be given by $\triangle_{+}^{1}$ and $\triangle_{+}^{2}$. By Section 2.6, there exists an interior automorphism $s \in \operatorname{Int}(\mathfrak{u})$ such that $s \triangle_{+}^{2}=\triangle_{+}^{1}$. Again replacing $\mathfrak{g}_{2}$ by $s . \mathfrak{g}_{2}$ and repeating above argument, we may assume that $\triangle_{+}^{1}=\triangle_{+}^{2}=\triangle_{+}$from the outset.

Using the conjugacy of the compact real forms of $\mathfrak{g}$ we construct in this case,

$$
\begin{aligned}
\mathfrak{u}=\sum_{\alpha \in \AA} \mathbb{R} i H_{\alpha} \oplus \sum_{\gamma \in \Delta^{\mathrm{re}}} \mathbb{R} i\left(e_{\gamma}+f_{\gamma}\right) \oplus \sum_{\gamma \in \Delta^{\mathrm{re}}} \mathbb{R}\left(e_{\gamma}-f_{\gamma}\right) \oplus \mathbb{R} i c \oplus \mathbb{R} i d \\
\oplus \sum_{\alpha \in \AA_{l}} \mathbb{R} i\left(H_{\alpha} t^{k n}+H_{\alpha} t^{-k n}\right) \oplus \sum_{\alpha \in \AA_{l}} \mathbb{R}\left(H_{\alpha} t^{k n}-H_{\alpha} t^{-k n}\right) \\
\oplus\left(\bigoplus_{j=0}^{k-1}\left(\sum_{\alpha \in \AA_{s}} \mathbb{R} i\left(h_{\alpha}^{j} t^{k n+j}+h_{\alpha}^{j} t^{-k n-j}\right) \oplus \sum_{\alpha \in \AA_{s}} \mathbb{R}\left(h_{\alpha}^{j} t^{k n+j}-h_{\alpha}^{j} t^{-k n-j}\right)\right)\right),
\end{aligned}
$$

where for $\gamma \in \triangle^{\text {re }}$,

$$
\begin{array}{llll}
e_{\gamma}=E_{\alpha, k s} & \text { and } & f_{\gamma}=E_{-\alpha,-k s} & \text { for } \alpha \in \AA_{l} \text { and } s \in \mathbb{Z}, \\
e_{\gamma}=E_{\alpha, k s+j} & \text { and } & f_{\gamma}=E_{-\alpha,-k s-j} & \text { for } \alpha \in \AA_{s}, s \in \mathbb{Z}, \text { and } j \in \mathbb{Z}_{k} .
\end{array}
$$

Here

$$
\begin{array}{ll}
E_{\alpha, k s}, E_{-\alpha,-k s}, H_{\alpha} & \text { for } \alpha \in \AA_{l}, \\
E_{\alpha, k s+j}, E_{-\alpha,-k s-j} h_{\alpha}^{j} & \text { for } \alpha \in \AA_{s} \text { and } j \in \mathbb{Z}_{k}
\end{array}
$$

are defined as in Section 2.5. 
Case 1: Suppose $\mathfrak{h}_{\mathbb{R}}$ has no $\sigma$-complex roots. Since the Vogan diagrams for $\mathfrak{g}_{1}$ and $\mathfrak{g}_{2}$ are the same, the automorphisms of $\Delta_{+}$defined by $\varpi_{1}$ and $\varpi_{2}$ have the same effect on $\mathfrak{h}^{*}$. Thus

$$
\begin{aligned}
\varpi_{1}(H) & =\varpi_{2}(H) \quad \text { for all } H \in \mathfrak{h}, \\
\varpi_{1}\left(\triangle^{\mathrm{im}}\right) & =\varpi_{2}\left(\triangle^{\mathrm{im}}\right),
\end{aligned}
$$

If $\alpha$ is a simple $\sigma$-imaginary real root, then

$$
\varpi_{1}\left(e_{j}\right)= \begin{cases}e_{j}=\varpi_{2}\left(e_{j}\right) & \text { if the } j \text {-th vertex is unpainted } \\ -e_{j}=\varpi_{2}\left(e_{j}\right) & \text { if the } j \text {-th vertex is painted. }\end{cases}
$$

Since $\mathfrak{h}$ and $\left\{e_{j}, f_{j}, j \in[0, l]\right\}$ generate $\mathfrak{g}$, it follows that $\varpi_{1}=\varpi_{2}$ on $\mathfrak{u}$; hence $\mathfrak{k}_{1}=\mathfrak{u}^{\varpi_{1}}=\mathfrak{k}_{2}$, and if $\mathfrak{p}_{j}$ for $j=1,2$ denotes the -1 eigenspace of $\varpi_{j}$ on $\mathfrak{u}$, then $\mathfrak{p}_{1}=\mathfrak{p}_{2}$. Hence

$$
\mathfrak{g}_{1}=\mathfrak{k}_{1} \oplus i \mathfrak{p}_{1}=\mathfrak{k}_{2} \oplus i \mathfrak{p}_{2}=\mathfrak{g}_{2} .
$$

Case 2: Suppose there exist $\sigma$-complex simple real roots in $\mathfrak{g}_{1}$ and $\mathfrak{g}_{2}$. Let $\rho$ denote the diagram automorphism of $S(A)$. In this case, the $\sigma$-imaginary roots are treated as in Case 1. If for all $\sigma$-complex roots $\gamma \in \triangle^{\text {re }}$, there exist $X_{\gamma} \in \mathfrak{g}_{\gamma}, \quad X_{\rho \gamma} \in \mathfrak{g}_{\rho \gamma}$ and constants $a_{\gamma}, b_{\gamma}$ such that

$$
\varpi_{1}\left(X_{\gamma}\right)=a_{\gamma} X_{\rho \gamma} \quad \text { and } \quad \varpi_{2}\left(X_{\gamma}\right)=b_{\gamma} X_{\rho \gamma},
$$

then the same calculations as those in [Batra 2000, Theorem 5.2, Case 2] show that for each pair $\gamma, \varpi \gamma$ of $\sigma$-complex simple roots, square roots $a_{\gamma}^{1 / 2}, a_{\rho \gamma}^{1 / 2}, b_{\gamma}^{1 / 2}$ and $b_{\rho \gamma}^{1 / 2}$ can be chosen such that $a_{\gamma}^{1 / 2} a_{\rho \gamma}^{1 / 2}=1$ and $b_{\gamma}^{1 / 2} b_{\rho \gamma}^{1 / 2}=1$. Then defining $H, H^{\prime} \in \mathfrak{u} \cap \mathfrak{h}$ such that $\alpha(H)=\alpha\left(H^{\prime}\right)=0$ for $\alpha$ a $\sigma$-imaginary simple root and

$$
\begin{aligned}
\exp \left(\frac{1}{2} \gamma(H)\right) & =a_{\gamma}{ }^{1 / 2}, & \exp \left(\frac{1}{2} \rho \gamma(H)\right) & =a_{\rho \gamma}^{1 / 2}, \\
\exp \left(\frac{1}{2} \gamma\left(H^{\prime}\right)\right) & =b_{\gamma}{ }^{1 / 2}, & \exp \left(\frac{1}{2} \rho \gamma\left(H^{\prime}\right)\right) & =b_{\rho \gamma}^{1 / 2}
\end{aligned}
$$

for $\gamma$ and $\rho \gamma$ both $\sigma$-complex simple roots, calculations similar to those in [Batra 2000, Theorem 5.2, Case 2] show that applying the identity

$$
\varpi_{2} \circ \operatorname{Ad}\left(\exp \frac{1}{2}\left(H-H^{\prime}\right)\right)=\operatorname{Ad}\left(\exp \frac{1}{2}\left(H-H^{\prime}\right)\right) \circ \varpi_{1}
$$

to the $\sigma$-eigenspaces of $\mathfrak{g}_{1}$ gives an isomorphism between $\mathfrak{g}_{1}$ and $\mathfrak{g}_{2}$.

Thus to complete the proof of the theorem we need to show the existence of $X_{\gamma} \in \mathfrak{g}_{\gamma}$ and $X_{\rho \gamma} \in \mathfrak{g}_{\rho \gamma}$, and constants $a_{\gamma}$ and $b_{\gamma}$ satisfying (4-5). Observe from Table 1 that for $\mathfrak{g}$ of type Aff $k$, with $k \neq 1, \sigma$-complex roots exist only when $\mathfrak{g}$ is of type $A_{2 \ell-1}^{(2)}$ or $D_{\ell+1}^{(2)}$. If $\gamma=\alpha+k s \delta \in \triangle^{\text {re }}$ for $\alpha \in \triangle_{l}$, then $\mathfrak{g}_{\gamma}=\mathbb{C} e_{\alpha} \otimes t^{k s}$, where $e_{\alpha} \in(\dot{\mathfrak{g}})_{\alpha}$. Since $e_{\alpha} \otimes t^{k s} \in \mathfrak{l}(\dot{\mathfrak{g}}, \mathrm{Id}, 1)$, (4-5) is satisfied in this case by [Batra 2000, 
Theorem 5.2, Case 2]. However, problems can arise if a $\sigma$-complex root $\gamma \in \Delta^{\text {re }}$ is a short root. Note that the following are the only short $\sigma$-complex simple roots:

$$
\begin{array}{ll}
\alpha_{0}, \alpha_{1}=\alpha_{\rho 0} & \text { when } \mathfrak{g} \text { is of type } A_{2 \ell-1}^{(2)}, \\
\alpha_{0}, \alpha_{\ell}=\alpha_{\rho 0} & \text { when } \mathfrak{g} \text { is of type } D_{\ell+1}^{(2)} .
\end{array}
$$

Let $H_{\rho_{(0)}}:=\exp (i \pi / 2) \operatorname{ad}\left(\stackrel{\circ}{p}_{\rho_{(0)}}\right)$. Then $\mathfrak{l}\left(\dot{\mathfrak{g}}, \mu H_{\rho_{(0)}}, \varepsilon_{4}\right)$ is a realization of $\mathfrak{g}$ adapted to the involution $\sigma$, and has a 2-element orbit. If $\mathfrak{g}_{\alpha_{0}}=\mathbb{C} X_{\alpha_{0}}$ and $\mathfrak{g}_{\rho\left(\alpha_{0}\right)}=\mathbb{C} X_{\alpha_{j}}$ for $\alpha_{j}$ a simple short root of $\mathfrak{g}$, then in the realization $\mathfrak{l}\left(\dot{\mathfrak{g}}, \mu H_{\rho_{(0)}}, \varepsilon_{4}\right)$ we have

$$
\begin{aligned}
& X_{\alpha_{0}}=\left(e_{-\theta^{0}}-e_{-\mu \theta^{0}}\right) \otimes t \text { for } \mathfrak{g} \text { of type } D_{\ell+1}^{(2)} \text { and } A_{2 \ell-1}^{(2)} \\
& X_{\alpha_{j}}=\left(e_{\dot{\alpha}_{j}}+e_{-\mu \dot{\alpha}_{j}}\right) \otimes t \quad \text { where } j=\ell \text { for } \mathfrak{g} \text { of type } D_{\ell+1}^{(2)}, \dot{\alpha}_{j} \in \triangle\left(D_{\ell+1}\right), \\
& j=1 \text { for } \mathfrak{g} \text { of type } A_{2 \ell-1}^{(2)}, \dot{\alpha}_{j} \in \triangle\left(A_{2 \ell-1}\right) .
\end{aligned}
$$

Since for appropriate $j$ (as explained above), $\varpi_{1}\left(\mathfrak{g}_{\alpha_{0}}\right) \subset \mathfrak{g}_{\alpha_{j}}$ and $\varpi_{2}\left(\mathfrak{g}_{\alpha_{0}}\right) \subset \mathfrak{g}_{\alpha_{j}}$, the equation above implies there exists constants $a_{\theta^{0}}^{1}, a_{\theta^{0}}^{2}, a_{\mu \theta^{0}}^{1}, a_{\mu \theta^{0}}^{2}$ and $b_{\theta^{0}}^{1}, b_{\theta^{0}}^{2}$, $b_{\mu \theta^{0}}^{1}, b_{\mu \theta^{0}}^{2}$ such that

$$
\begin{aligned}
\varpi_{1}\left(e_{-\theta^{0}} \otimes t\right) & =a_{\theta^{0}}^{1} e_{\dot{\alpha}_{j}} \otimes t+a_{\mu \theta^{0}}^{1} e_{\mu \dot{\alpha}_{j}} \otimes t, \\
\varpi_{1}\left(e_{-\mu \theta^{0}} \otimes t\right) & =a_{\theta^{0}}^{2} e_{\dot{\alpha}_{j}} \otimes t+a_{\mu \theta^{0}}^{2} e_{\mu \dot{\alpha}_{j}} \otimes t, \\
\varpi_{2}\left(e_{-\theta^{0}} \otimes t\right) & =b_{\theta^{0}}^{1} e_{\dot{\alpha}_{j}} \otimes t+b_{\mu \theta^{0}}^{1} e_{\mu \dot{\alpha}_{j}} \otimes t, \\
\varpi_{2}\left(e_{-\mu \theta^{0}} \otimes t\right) & =b_{\theta^{0}}^{2} e_{\dot{\alpha}_{j}} \otimes t+b_{\mu \theta^{0}}^{2} e_{\mu \dot{\alpha}_{j}} \otimes t .
\end{aligned}
$$

We claim $a_{\theta^{0}}^{1}-a_{\theta^{0}}^{2}=a_{\mu \theta^{0}}^{1}-a_{\mu \theta^{0}}^{2}$ and $b_{\theta^{0}}^{1}-b_{\theta^{0}}^{2}=b_{\mu \theta^{0}}^{1}-b_{\mu \theta^{0}}^{2}$.

Proof of the claim. Recall that $\varpi_{1}$ and $\varpi_{2}$ are restrictions of the involution $\sigma$ to the compact forms $\mathfrak{u}_{1}$ and $\mathfrak{u}_{2}$ adapted to $\mathfrak{g}_{1}$ and $\mathfrak{g}_{2}$, respectively. Since for $j=1,2$, the Cartan involution $\sigma$ is adapted to both of the $\mathfrak{g}_{j}$, and since the realization $\mathfrak{l}\left(\dot{\mathfrak{g}}, \mu H_{\rho_{(0)}}, \varepsilon_{4}\right)$ of $\mathfrak{g}$ is adapted to $\left(\mathfrak{g}_{j}, \sigma, \mathfrak{h}\right)$, by [Ben Messaoud and Rousseau 2003, Proposition 3.5] there exist $\dot{\sigma}_{j} \in \operatorname{Aut}(\dot{\mathfrak{g}})$ that commute with $\mu H_{\rho_{(0)}}$ and are such that $\varpi_{j}=\dot{\sigma}_{j} \otimes 1$ on the compact form $\mathfrak{u}$ of $\mathfrak{l}\left(\dot{\mathfrak{g}}, \mu H_{\rho_{(0)}}, \varepsilon_{4}\right)$.

Since $\mu H_{\rho_{(0)}}\left(e_{\mu \theta^{0}} \otimes t\right)=-i e_{\theta^{0}} \otimes t$, we have $e_{\theta^{0}} \otimes t=i \mu H_{\rho_{(0)}}\left(e_{\mu \theta^{0}} \otimes t\right)$. Hence

$$
\begin{aligned}
a_{\theta^{0}}^{2} g e_{\dot{\alpha}_{j}} \otimes & t+a_{\mu \theta^{0}}^{2} e_{\mu \dot{\alpha}_{j}} \otimes t=\varpi_{1}\left(e_{-\mu \theta^{0}} \otimes t\right)=\varpi_{1}\left(i \mu H_{\rho_{(0)}}\left(e_{-\theta^{0}}\right) \otimes t\right) \\
= & \dot{\sigma}_{1}\left(i\left(\mu H_{\rho_{(0)}}\left(e_{-\theta^{0}}\right)\right) \otimes t=i g \mu H_{\rho_{(0)}}\left(\dot{\sigma}_{1}\left(e_{-\theta^{0}}\right)\right) \otimes t\right. \\
& =i g \mu H_{\rho_{(0)}}\left(\left(\dot{\sigma}_{1}\left(e_{-\theta^{0}}\right)\right) \otimes t\right)=i g \mu H_{\rho_{(0)}}\left(\left(\varpi_{1}\left(e_{-\theta^{0}} \otimes t\right)\right)\right. \\
& =i g \mu H_{\rho_{(0)}}\left(a_{\theta^{0}}^{1} e_{\dot{\alpha}_{j}} \otimes t+a_{\mu \theta^{0}}^{1} g e_{\mu \dot{\alpha}_{j}} \otimes t\right) \\
& =i^{2} g\left(a_{\theta^{0}}^{1} e_{\mu \dot{\alpha}_{j}} \otimes t+a_{\mu \theta^{0}}^{1} g e_{\dot{\alpha}_{j}} \otimes t\right)=-a_{\theta^{0}}^{1} e_{\mu \dot{\alpha}_{j}} \otimes t-a_{\mu \theta^{0}}^{1} g e_{\dot{\alpha}_{j}} \otimes t .
\end{aligned}
$$

Comparing coefficients, we get $a_{\theta^{0}}^{2}=-a_{\mu \theta^{0}}^{1}$ and $a_{\mu \theta^{0}}^{2}=-a_{\theta^{0}}^{1}$. Hence $a_{\theta^{0}}^{1}-a_{\theta^{0}}^{2}=$ $-a_{\mu \theta^{0}}^{2}+a_{\mu \theta^{0}}^{1}$ as desired. It can be similarly shown that $b_{\theta^{0}}^{1}-b_{\theta^{0}}^{2}=-b_{\mu \theta^{0}}^{2}+b_{\mu \theta^{0}}^{1}$. 
Thus for short $\sigma$-complex simple roots, there exist $X_{\alpha_{0}} \in \mathfrak{g}_{\alpha_{0}}$ and $X_{\alpha_{j}} \in \mathfrak{g}_{\rho \alpha_{0}}$ such that (4-5) is satisfied. Hence the theorem.

Using the definition of equivalence relations between the Vogan diagrams (see Section 4.4) and [Knapp 1996a, Figures 6.1 and 6.2], we give in the following table the nonequivalent Vogan diagrams of the twisted affine Kac-Moody Lie algebras corresponding to nontrivial involutions of first type. Note that owing to the equivalence relation as described in Example 4.6, the nonequivalent Vogan diagrams for $\mathfrak{g}$ correspond to the following involutions of first type: $\mu \otimes 1$, $\exp i \pi \operatorname{ad}\left(p_{i}\right)$ for $1 \leq i \leq l / 2$ and $\exp i \pi \operatorname{ad}\left(\stackrel{\circ}{p}_{i}\right)$ for $1 \leq i \leq l$. Likewise, by the equivalence relations described in Examples 4.7 and 4.9, the nonequivalent Vogan diagrams for $\mathfrak{g}$ correspond to the following involutions of first type: $\mu \otimes 1$, $\exp i \pi \operatorname{ad}\left(p_{l}\right), \quad \exp i \pi \operatorname{ad}\left(\stackrel{\circ}{p}_{l}\right)$, and $\exp i \pi \operatorname{ad}\left(\dot{p}_{j}\right)$ for $1 \leq j \leq l / 2$, and by the equivalence relations of the kind described in Example 4.8, the nonequivalent Vogan diagrams for $\mathfrak{g}$ correspond to the following involutions of first type: $\rho$, $\rho\left(\mu^{2} \exp i \pi \operatorname{ad}\left(\stackrel{\circ}{p}_{1}\right) \otimes 1\right) \exp i \pi \operatorname{ad}\left(\stackrel{\circ}{p}_{l}\right)$, and $\rho \exp i \pi \operatorname{ad}\left(\stackrel{\circ}{p}_{j}\right)$ for $1 \leq j \leq(l+1) / 2$. The nonequivalent Vogan diagrams for the twisted affine Kac-Moody Lie algebras of type $A_{2 l}^{(2)}, D_{2 r+1}^{(2)}$ for $r \geq 1, D_{2 r}^{(2)}$ for $r \geq 2, E_{6}^{(2)}$ and $D_{4}^{(2)}$ are similarly studied.

It can be easily observed from Tables 2 and 3 that the number of Vogan diagrams corresponding to nontrivial involutions of first type is equal to the number of almost compact noncompact real forms of twisted affine Kac-Moody Lie algebras as given in [Ben Messaoud and Rousseau 2003], thereby suggesting the existence of a bijection between the equivalence classes of the Vogan diagrams and the isomorphism classes of the almost compact real forms of twisted affine Kac-Moody Lie algebras.

\section{Acknowledgment}

I thank Dr. Punita Batra for suggesting the problem and for providing me with the references [Bausch 1989] and [Rousseau 1989].

\section{References}

[Back-Valente et al. 1995] V. Back-Valente, N. Bardy-Panse, H. Ben Messaoud, and G. Rousseau, "Formes presque-déployées des algèbres de Kac-Moody: Classification et racines relatives", J. Algebra 171:1 (1995), 43-96. MR 96d:17022 Zbl 0823.17034

[Batra 2000] P. Batra, "Invariants of real forms of affine Kac-Moody Lie algebras", J. Algebra 223:1 (2000), 208-236. MR 2001g:17033 Zbl 0958.17009

[Batra 2002] P. Batra, "Vogan diagrams of real forms of affine Kac-Moody Lie algebras", J. Algebra 251:1 (2002), 80-97. MR 2003d:17027 Zbl 1026.17027

[Bausch 1989] J. Bausch, "Étude et classification des automorphismes d'ordre fini et de première espèce des algèbres de Kac-Moody affines", pp. 5-124 in Algèbres de Kac-Moody affines, Inst. Élie Cartan 11, Univ. Nancy, 1989. MR 1021053 Zbl 0698.17016 


\begin{tabular}{|c|c|c|}
\hline $\mathfrak{g}$ & e-Vogan diagram & involution of first type \\
\hline$A_{2}^{(2)}$ & 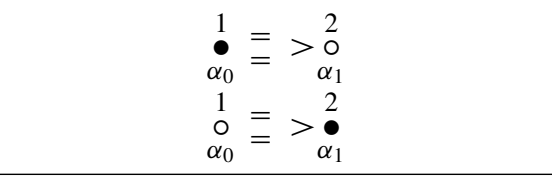 & $\begin{array}{l}\mu \otimes 1 \\
\exp i \pi \operatorname{ad}\left(\stackrel{\circ}{p}_{1}\right)=\exp i \pi \operatorname{ad}\left(p_{1}\right)\end{array}$ \\
\hline$A_{2 l}^{(2)}$ & 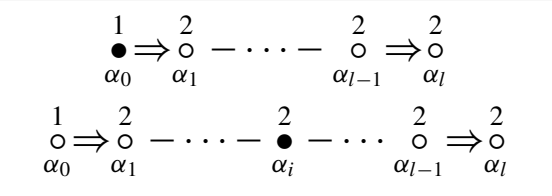 & $\begin{array}{l}\mu \otimes 1 \\
\exp i \pi \operatorname{ad}\left(\stackrel{\circ}{p}_{i}\right)=\exp i \pi \operatorname{ad}\left(p_{i}\right) \\
\text { for } 1 \leq i \leq l\end{array}$ \\
\hline $\begin{array}{l}A_{2 l-1}^{(2)} \\
\text { for } l \geq 3\end{array}$ & 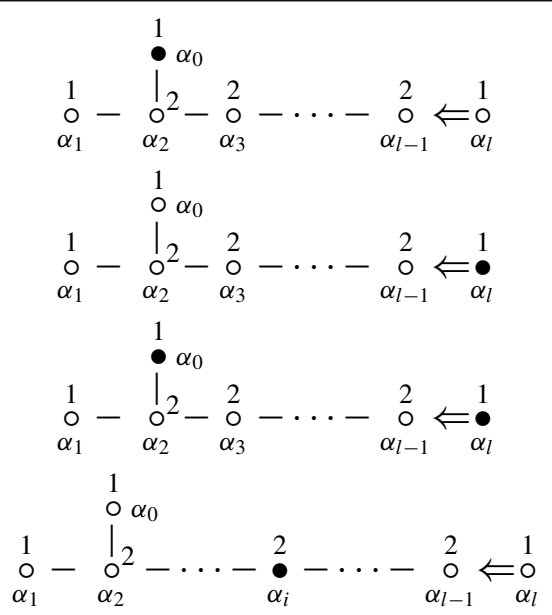 & $\begin{array}{l}(\mu \otimes 1) \exp i \pi \operatorname{ad}\left(\stackrel{\circ}{p}_{l}\right) \\
\quad=\exp i \pi \operatorname{ad}\left(p_{l}\right)\end{array}$ \\
\hline
\end{tabular}

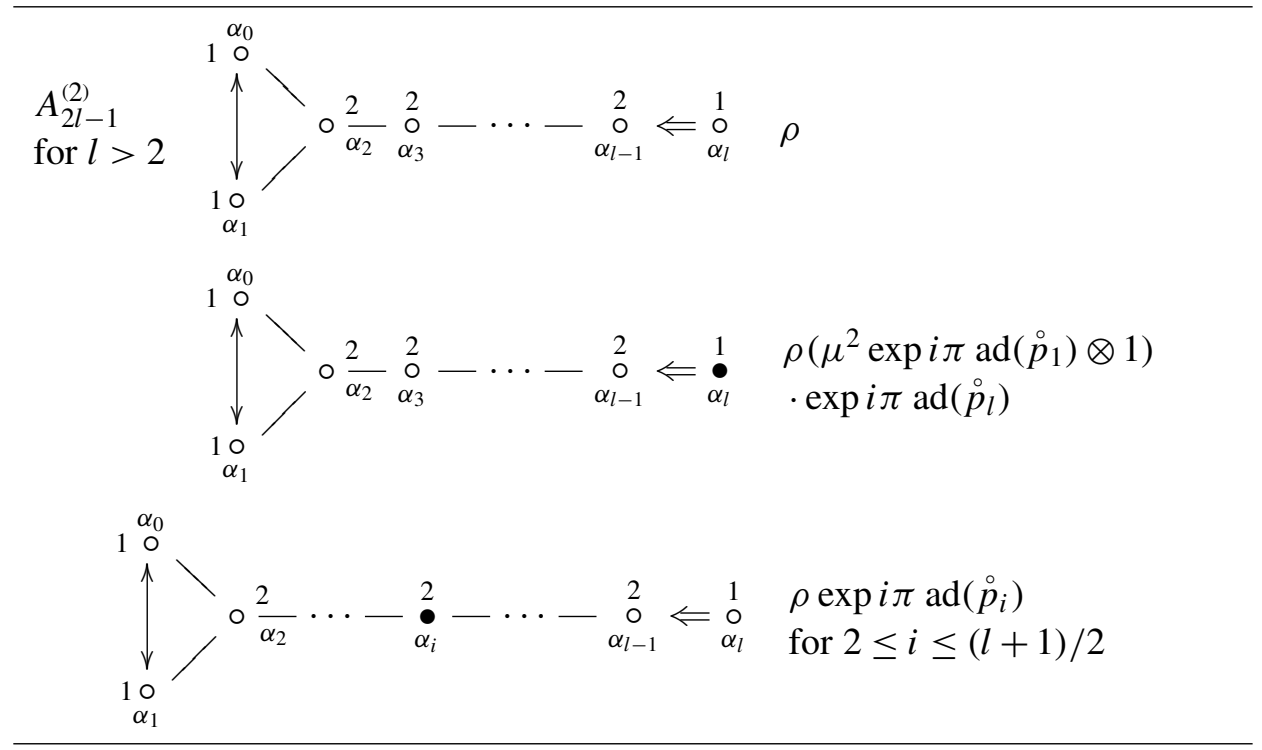

Table 2. Vogan diagrams for affine Kac-Moody Lie algebras of type Aff 2. 


\begin{tabular}{|c|c|c|}
\hline $\mathfrak{g}$ & e-Vogan diagram & involution of first type \\
\hline \multirow{3}{*}{$\begin{array}{l}D_{l+1}^{(2)} \\
\text { for } l \geq 2\end{array}$} & 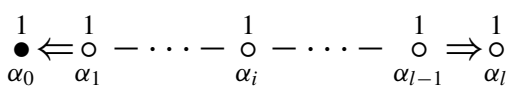 & $\mu \otimes 1$ \\
\hline & 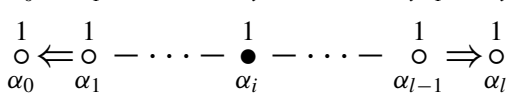 & $\begin{array}{l}\exp i \pi \operatorname{ad}\left(\stackrel{\circ}{p}_{i}\right) \mu \otimes 1= \\
\exp i \pi \operatorname{ad}\left(p_{i}\right) \text { for } 1 \leq i \leq l / 2\end{array}$ \\
\hline & 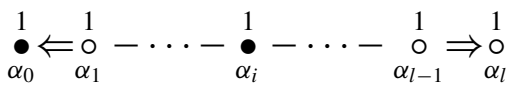 & $\exp i \pi \operatorname{ad}\left(\stackrel{\circ}{p}_{i}\right)$ for $1 \leq i \leq l$ \\
\hline \multirow[t]{2}{*}{$\begin{array}{l}D_{2 r+1}^{(2)} \\
\text { for } r \geq 1\end{array}$} & 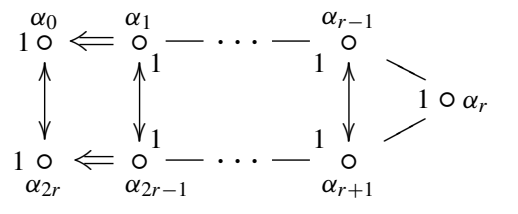 & $\rho$ \\
\hline & 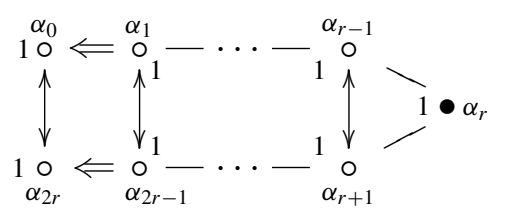 & $\begin{array}{l}\rho \exp i \pi \operatorname{ad}\left(p_{r}\right)= \\
\rho \exp i \pi \operatorname{ad}\left(\stackrel{\circ}{p}_{r}\right) \\
\quad \cdot\left(\mu^{2} \exp i \pi \operatorname{ad}\left(\stackrel{\circ}{p}_{2} r\right) \otimes 1\right)\end{array}$ \\
\hline $\begin{array}{l}D_{2 r}^{(2)} \\
\text { for } r \geq 2\end{array}$ & 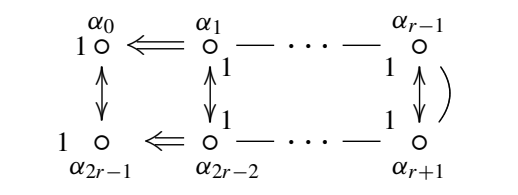 & $\rho$ \\
\hline \multirow[t]{4}{*}{$E_{6}^{(2)}$} & $\underset{\alpha_{0}}{1}-\underset{\alpha_{1}}{\bullet}-\underset{\alpha_{2}}{\stackrel{0}{\circ}} \Leftarrow \underset{\alpha_{3}}{\stackrel{2}{\circ}}-\underset{\alpha_{4}}{\stackrel{\circ}{\circ}}$ & $\begin{array}{l}\exp i \pi \operatorname{ad}\left(\stackrel{\circ}{p}_{1}\right) \\
\quad=\exp i \pi \operatorname{ad}\left(p_{1}\right)\end{array}$ \\
\hline & $\underset{\alpha_{0}}{\bullet}-\underset{\alpha_{1}}{\stackrel{2}{\circ}}-\underset{\alpha_{2}}{\stackrel{3}{\circ}} \underset{\alpha_{3}}{\stackrel{2}{\circ}}-\underset{\alpha_{4}}{\bullet}$ & $\exp i \pi \operatorname{ad}\left(\stackrel{\circ}{p}_{4}\right)$ \\
\hline & 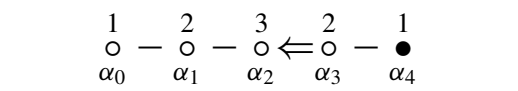 & $\begin{array}{l}(\mu \otimes 1) \exp i \pi \operatorname{ad}\left(\stackrel{\circ}{p}_{4}\right) \\
\quad=\exp i \pi \operatorname{ad}\left(p_{4}\right)\end{array}$ \\
\hline & 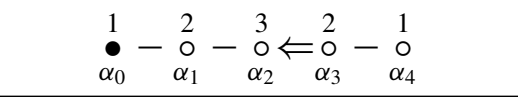 & $\mu \otimes 1$ \\
\hline$D_{4}^{(3)}$ & 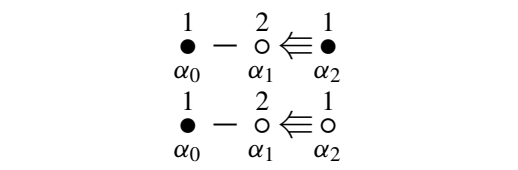 & $\begin{array}{l}\exp i \pi \operatorname{ad}\left(\stackrel{\circ}{p}_{2}\right) \\
\zeta^{3} \otimes 1\end{array}$ \\
\hline
\end{tabular}

Table 3. Vogan diagrams for affine Kac-Moody Lie algebras of type Aff 2 and 3 (continued). 
[Ben Messaoud and Rousseau 2007] H. Ben Messaoud and G. Rousseau, "Sous-algèbres de Cartan des algèbres de Kac-Moody affines réelles presque compactes”, J. Lie Theory 17:1 (2007), 1-25. MR 2008c:17017 Zbl 1121.17015

[Kac 1990] V. G. Kac, Infinite-dimensional Lie algebras, 3rd ed., Cambridge University Press, 1990. MR 92k:17038 Zbl 0716.17022

[Knapp 1996a] A. W. Knapp, Lie groups beyond an introduction, Progress in Mathematics 140, Birkhäuser, Boston, 1996. MR 98b:22002 Zbl 0862.22006

[Knapp 1996b] A. W. Knapp, “A quick proof of the classification of simple real Lie algebras", Proc. Amer. Math. Soc. 124:10 (1996), 3257-3259. MR 96m:17015 Zbl 0865.17002

[Moody and Pianzola 1995] R. V. Moody and A. Pianzola, Lie algebras with triangular decompositions, Wiley, New York, 1995. MR 96d:17025 Zbl 0874.17026

[Peterson and Kac 1983] D. H. Peterson and V. G. Kac, "Infinite flag varieties and conjugacy theorems”, Proc. Nat. Acad. Sci. U.S.A. 80:6 i. (1983), 1778-1782. MR 84g:17017 Zbl 0512.17008

[Rousseau 1989] G. Rousseau, "Formes réelles presque-compactes des algèbres de Kac-Moody affines”, pp. 175-205 in Algèbres de Kac-Moody affines, Inst. Élie Cartan 11, Univ. Nancy, 1989. MR 1021056 Zbl 0705.17016

Received July 15, 2008.

TANUSREe PAL

HARISH CHANDRA RESEARCH INSTITUTE

CHHATNAG ROAD

JHUNSI

AllahabAd 211019

INDIA

tanusree@hri.res.in 